\title{
Predicting the UV-VIS Spectra of Tetraarylcyclopentadienones: Using DFT Molecular Orbital Energies to Model Electronic Transitions
}

\author{
Robert G. Potter, Thomas S. Hughes* \\ Department of Chemistry, Cook Physical Sciences Building, 82 University Place, University of Vermont, \\ Burlington, VT 05401
}

\section{Supporting Information}

\section{Contents}

Full Reference 25

Correlation of Experimental UV/Vis and Computed Electronic Transition Energies

Spectra of Novel Compounds

General Experimental Details

Computational Details

\section{Full Reference 25}

Gaussian 03, Revision C.02, M. J. Frisch, G. W. Trucks, H. B. Schlegel, G. E. Scuseria, M. A. Robb, J. R. Cheeseman, J. A. Montgomery, Jr., T. Vreven, K. N. Kudin, J. C. Burant, J. M. Millam, S. S. Iyengar, J. Tomasi, V. Barone, B. Mennucci, M. Cossi, G. Scalmani, N. Rega, G. A. Petersson, H. Nakatsuji, M. Hada, M. Ehara, K. Toyota, R. Fukuda, J. Hasegawa, M. Ishida, T. Nakajima, Y. Honda, O. Kitao, H. Nakai, M. Klene, X. Li, J. E. Knox, H. P. Hratchian, J. B. Cross, V. Bakken, C. Adamo, J. Jaramillo, R. Gomperts, R. E. Stratmann, O. Yazyev, A. J. Austin, R. Cammi, C. Pomelli, J. W. Ochterski, P. Y. Ayala, K. Morokuma, G. A. Voth, P. Salvador, J. J. Dannenberg, V. G. Zakrzewski, S. Dapprich, A. D. Daniels, M. C. Strain, O. Farkas, D. K. Malick, A. D. Rabuck, K. Raghavachari, J. B. Foresman, J. V. Ortiz, Q. Cui, A. G. Baboul, S. Clifford, J. Cioslowski, B. B. Stefanov, G. Liu, A. Liashenko, P. Piskorz, I. Komaromi, R. L. Martin, D. J. Fox, T. Keith, M. A. Al-Laham, C. Y. Peng, A. Nanayakkara, M. Challacombe, P. M. W. Gill, B. Johnson, W. Chen, M. W. Wong, C. Gonzalez, and J. A. Pople, Gaussian, Inc., Wallingford CT, 2004. 


\section{Correlation of Experimental UV/Vis and Computed Electronic Transition $\underline{\text { Energies }}$}

\section{3-,4-Phenyl Substituted Tetraarylcyclopentadienones reported in the literature}

Graphs of tetraphenylcyclopentadienones (TPCPDs) substituted on the 3- and 4-phenyl positions showed a linear relationship with the B3LYP/6-31G(d) MO energies as do the compounds reported in the full paper. However, due to the fewer number of points and the use of different solvents for different TPCPDs we are not yet comfortable drawing firm conclusions from these results. The visible band, $\lambda^{3}$, of the TPCPDs does, however, seem to be associated with the same single electron HOMO-LUMO transition seen in compounds $\mathbf{1 a}-\mathbf{1 h}$. It should be noted that although all of the experimental transitions fit a good linear correlation with those computed, each individual transition has a small sampling of points and as a result, no consistent linear correlation exists for only the $\lambda^{1}, \lambda^{2}$, or $\lambda^{3}$ transitions.

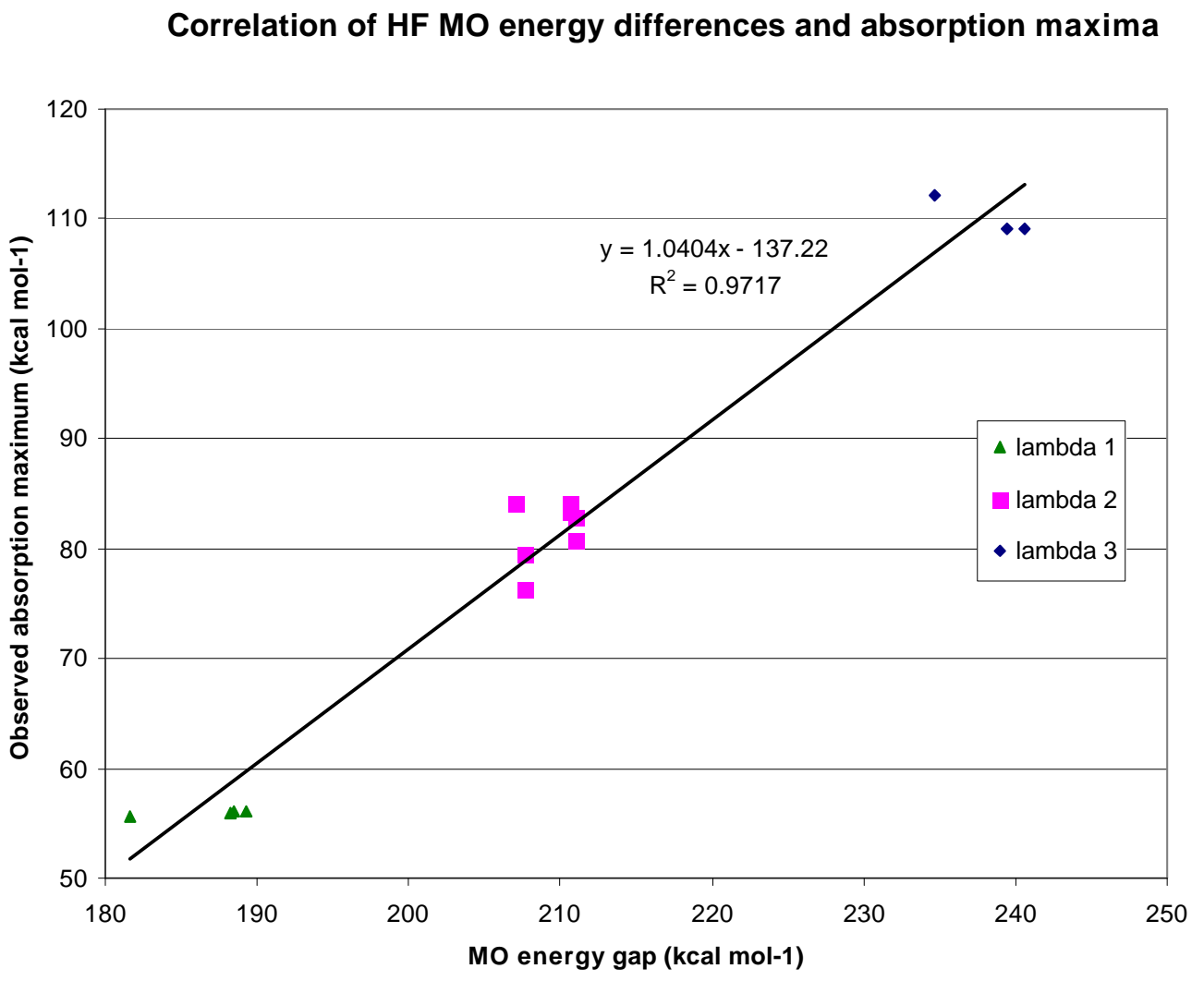

\section{Correlations for 1a - $1 \mathrm{~h}$ using HF/6-31G(d) and MP2/6-31G(d) MO methods}

HF and MP2 MOs were also generated, and the correlation of the relevant MO energy differences and the observed optical transitions is shown below, along with the linear leastsquares $\mathrm{R}^{2}$ errors. 

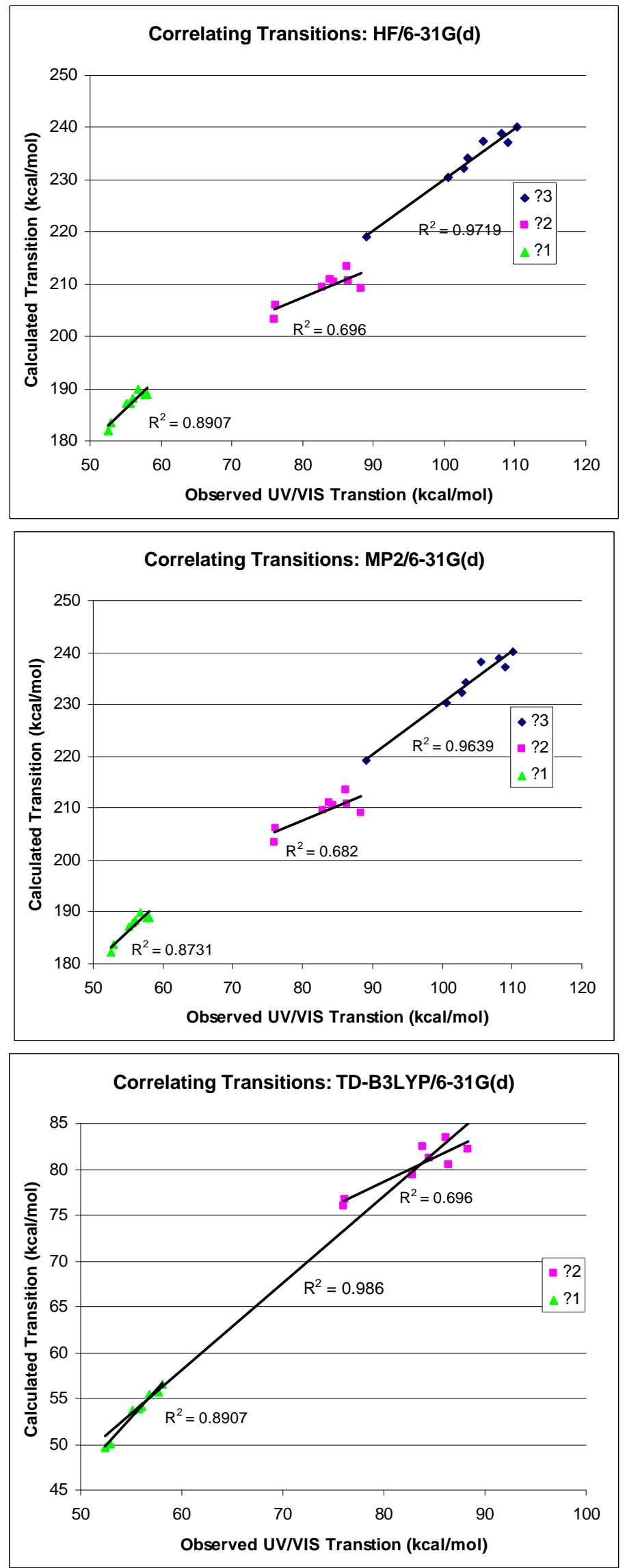


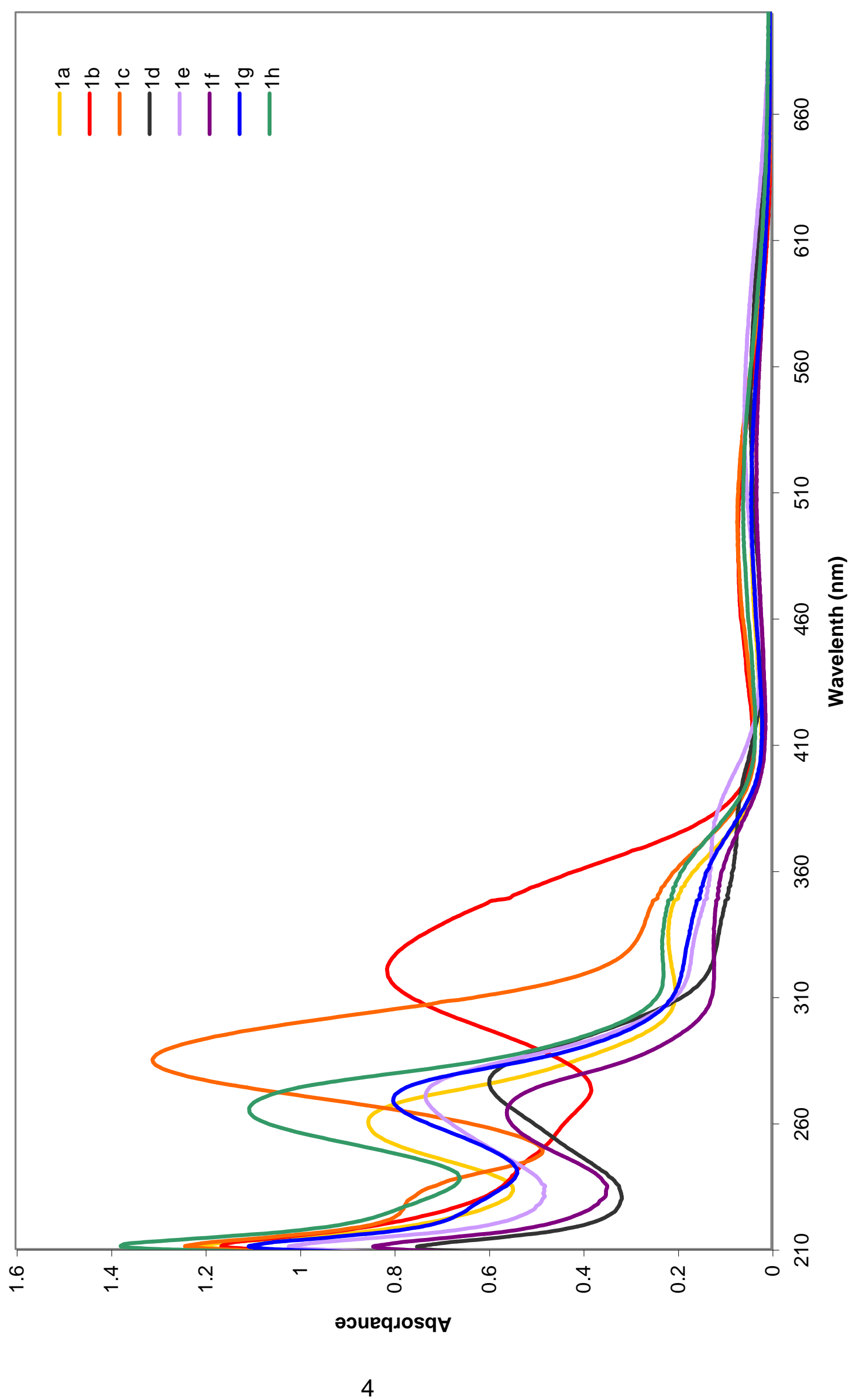




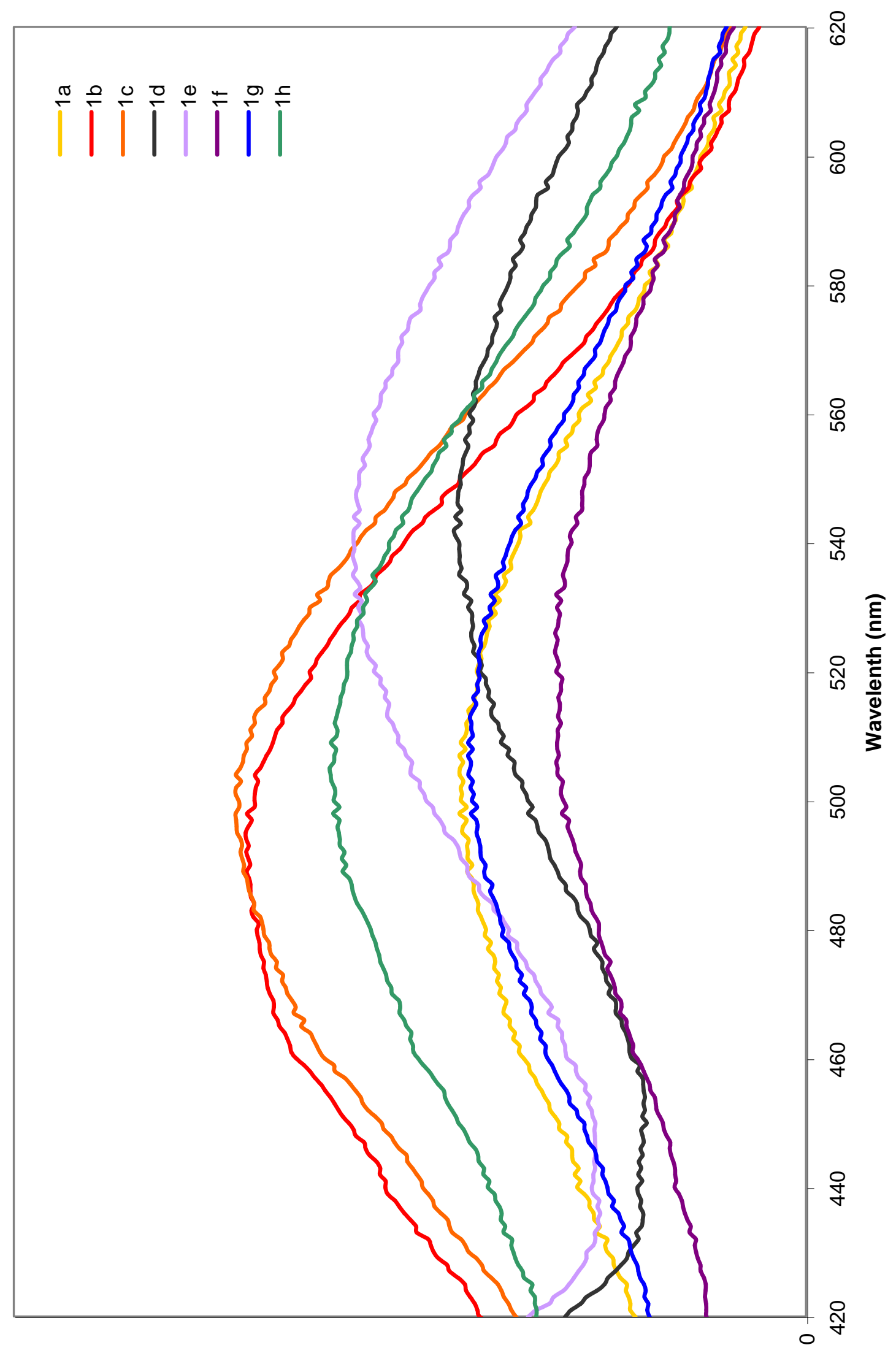

әэuеqлоsq 


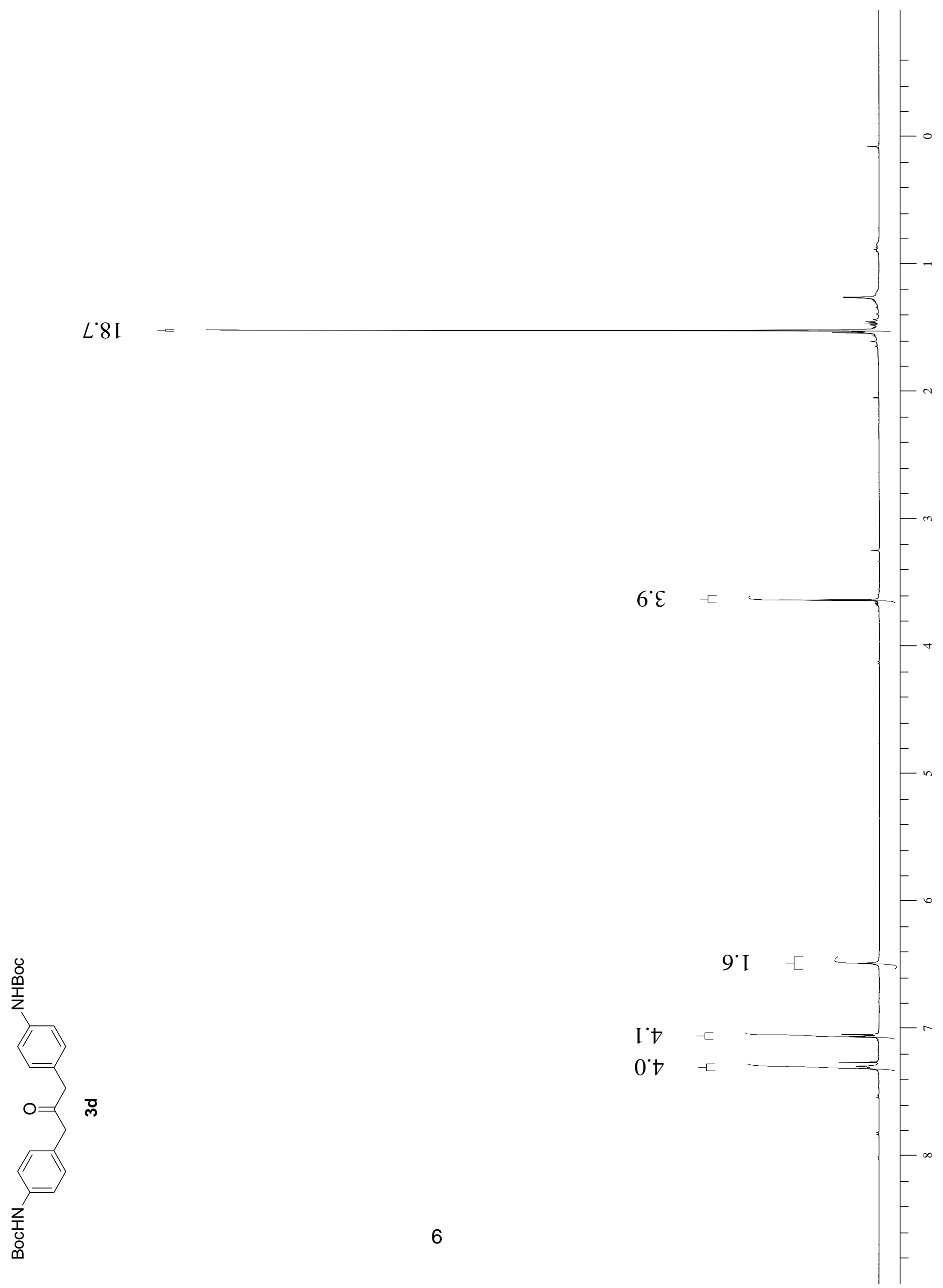




$$
\mid
$$


<smiles>[C+]c1ccc(C2=C(c3ccccc3)C(c3ccccc3)=C(c3ccc([O-])cc3)C2=O)cc1</smiles>

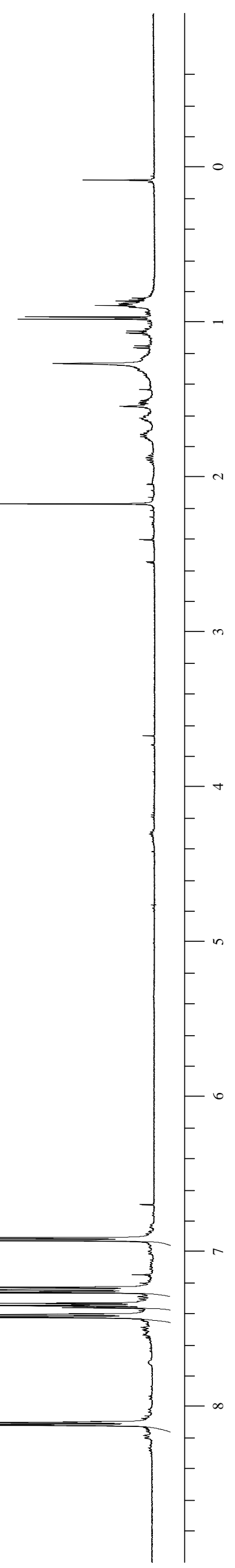


<smiles>O=C1C(c2ccccc2)=C(c2ccccc2)C(c2ccc([O-])cc2)=C1c1ccc([Co])cc1</smiles>

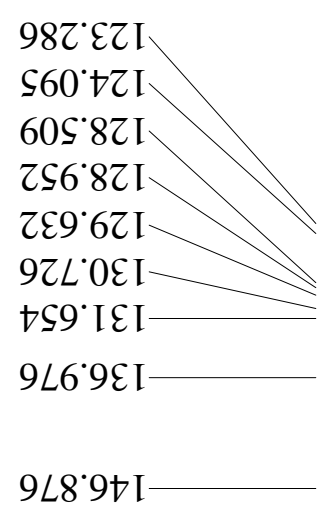

$687^{\circ} L S I$ 


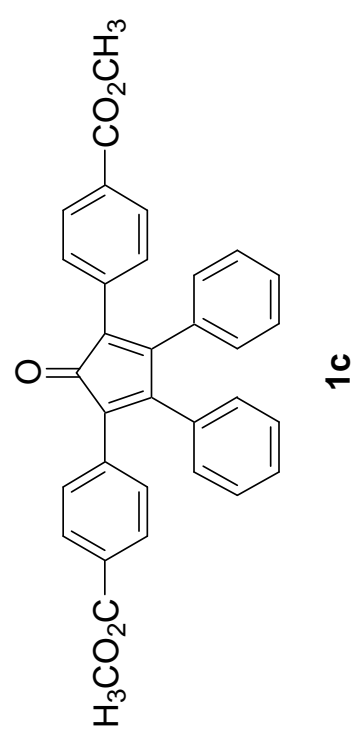

$0^{\circ} 9-\sqsubset$

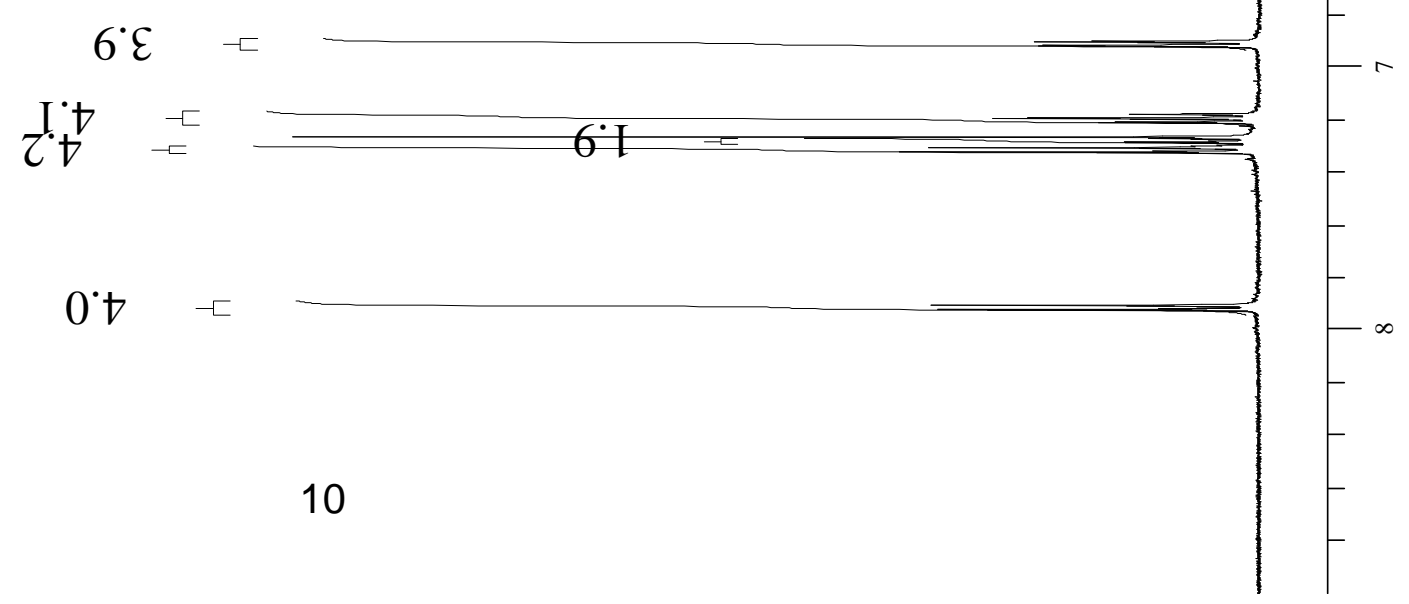




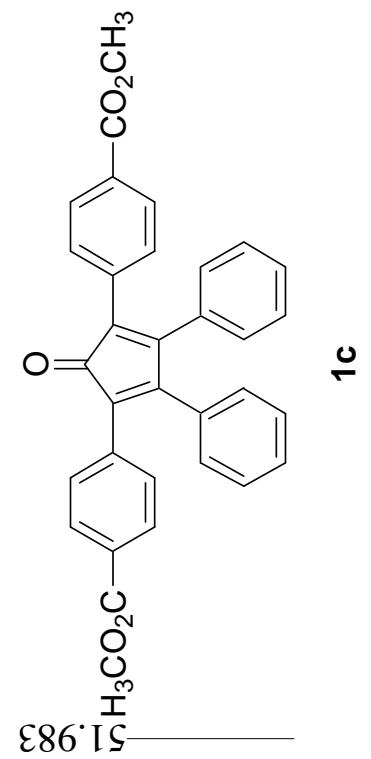

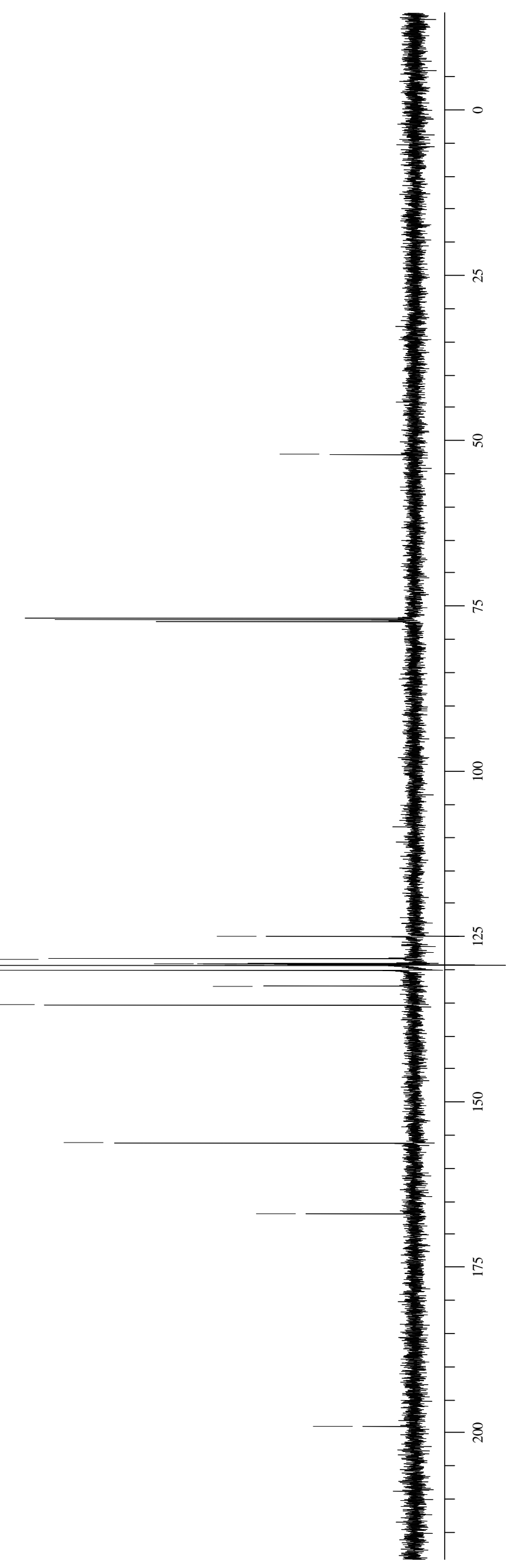




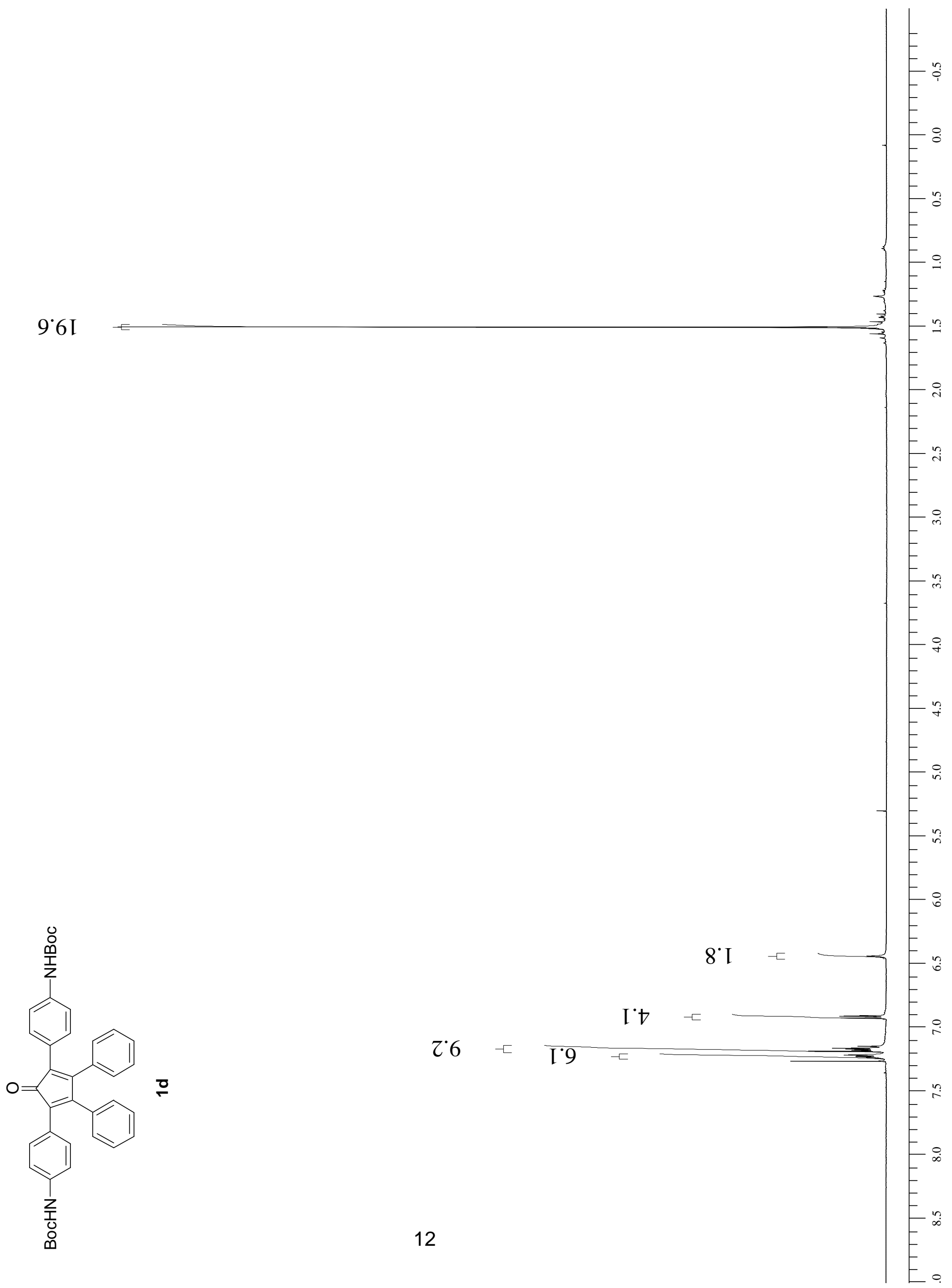




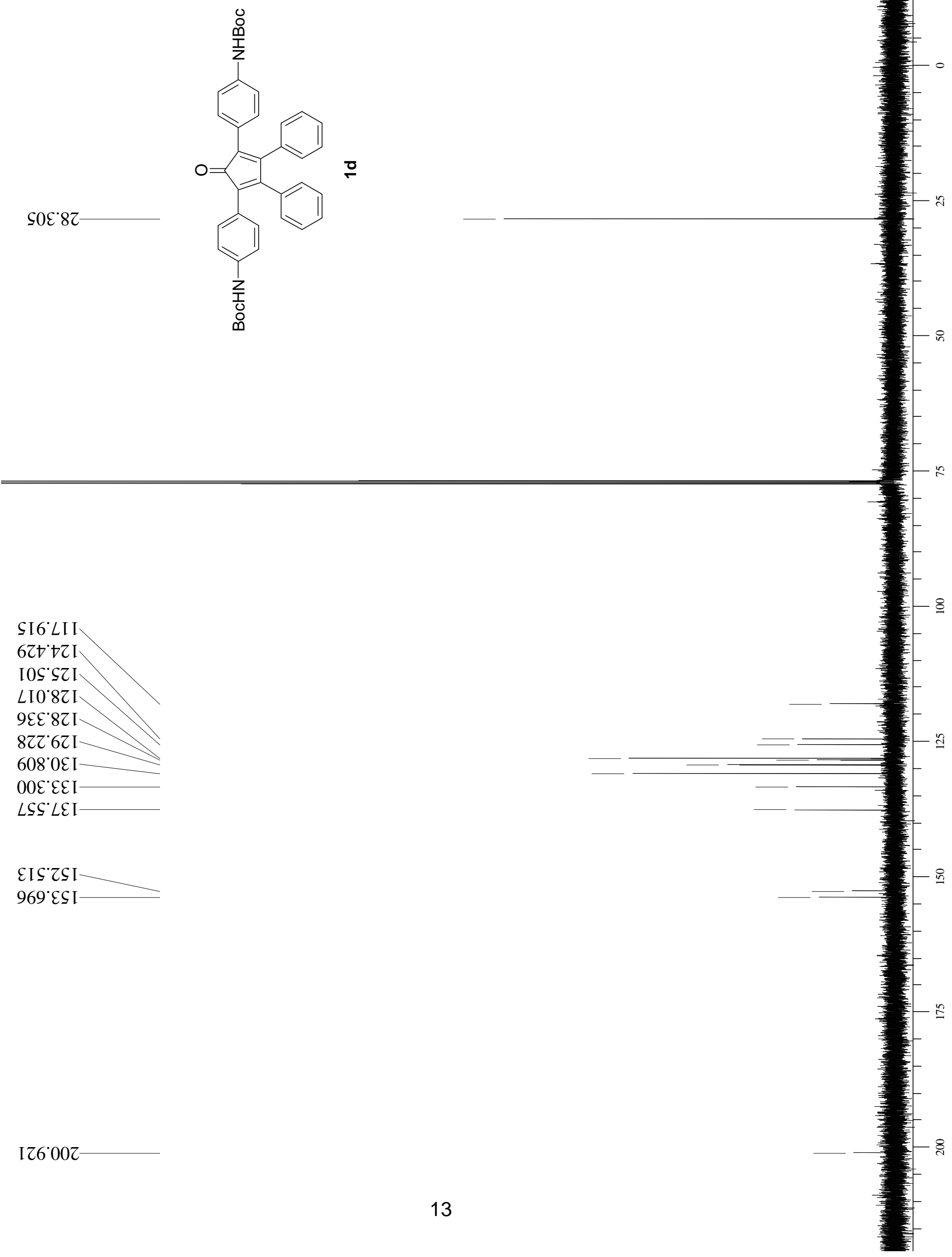




\section{$\underline{\text { General Experimental Details }}$}

All starting materials and solvents were used as received without further purification, unless otherwise noted. UV-VIS spectra were obtained in dioxane at room temperature. NMR spectra were recorded at 500 and $125 \mathrm{MHz}$ for ${ }^{1} \mathrm{H}$ and ${ }^{13} \mathrm{C}$ respectively at room temperature $(300 \mathrm{~K}+/-$ $1 \mathrm{~K})$ in $d-\mathrm{CHCl}_{3}$ unless otherwise indicated. IR spectra were obtained neat at room temperature unless otherwise indicated; IR spectra of solids were obtained using an ATR probe. Low resolution CI-MS were obtained with a direct insertion probe or on a GCMS using a 5\% phenyl column. Chromatography was carried out using $40 \mu \mathrm{l}$ silica gel. 


\section{$\underline{\text { Computational Details }}$}

Geometry Optimization and MO calculations of TACPDs

The Cartesian coordinates of the B3LYP/6-31G(d) geometry optimized TACPDs are listed below in their Gaussian input file format.

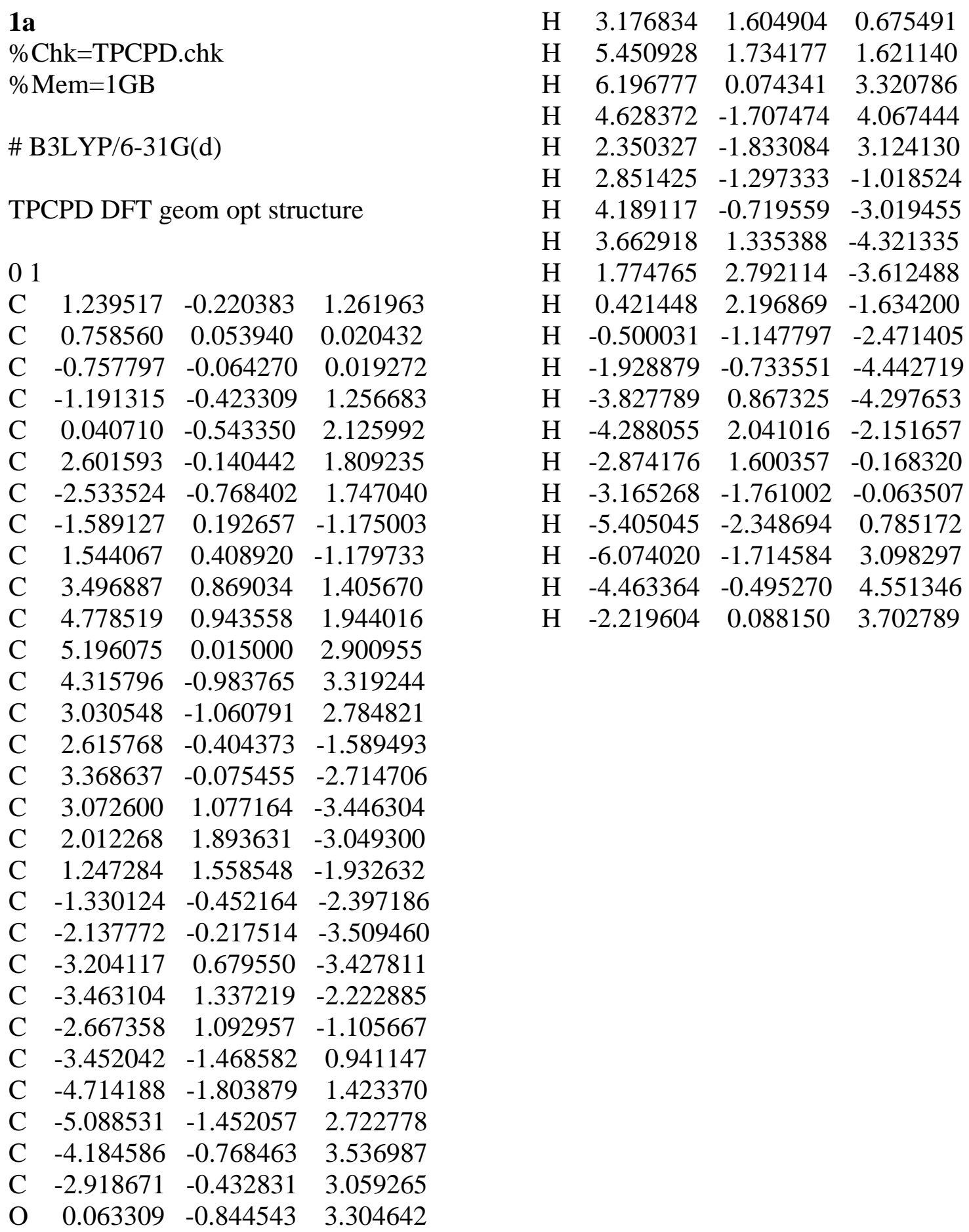




\begin{tabular}{|c|c|c|c|}
\hline & $\begin{array}{l}\mathrm{k}=\mathrm{NO} 2 \_\mathrm{N} \\
\mathrm{em}=1 \mathrm{~GB}\end{array}$ & NO2_TPCPI & D.chk \\
\hline & 3LYP/6-31 & $1 \mathrm{G}(\mathrm{d})$ & \\
\hline & NO2_TH & PCPD geom & n opt structure \\
\hline 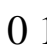 & & & \\
\hline $\mathrm{C}$ & -4.887569 & -0.848606 & 0.763761 \\
\hline $\mathrm{C}$ & -5.164773 & -2.028728 & 0.073625 \\
\hline $\mathrm{C}$ & -4.182867 & -2.700918 & -0.650457 \\
\hline $\mathrm{C}$ & -2.896463 & -2.174517 & -0.690353 \\
\hline $\mathrm{C}$ & -2.583077 & -0.974877 & -0.018779 \\
\hline $\mathrm{C}$ & -3.600698 & -0.330678 & 0.71548 \\
\hline $\mathrm{C}$ & -1.215608 & -0.442247 & -0.055811 \\
\hline $\mathrm{C}$ & -0.000018 & -1.337856 & -0.000144 \\
\hline $\mathrm{C}$ & 1.21558 & -0.442251 & 0.055602 \\
\hline $\mathrm{C}$ & 2.583049 & -0.974891 & 0.01864 \\
\hline $\mathrm{C}$ & 2.89632 & -2.174628 & 0.690104 \\
\hline $\mathrm{C}$ & 4.182714 & -2.701056 & 0.650311 \\
\hline $\mathrm{C}$ & 5.164734 & -2.028788 & -0.073547 \\
\hline $\mathrm{C}$ & 4.887653 & -0.848564 & -0.763551 \\
\hline $\mathrm{C}$ & 3.600786 & -0.330607 & -0.715375 \\
\hline $\mathrm{O}$ & -0.000013 & -2.554155 & -0.000177 \\
\hline $\mathrm{N}$ & -6.526311 & -2.577895 & 0.116566 \\
\hline $\mathrm{O}$ & -6.742429 & -3.623151 & -0.497341 \\
\hline $\mathrm{O}$ & -7.372783 & -1.959026 & 0.762998 \\
\hline $\mathrm{N}$ & 6.526267 & -2.577981 & -0.116377 \\
\hline $\mathrm{O}$ & 6.74228 & -3.62332 & 0.497425 \\
\hline $\mathrm{O}$ & 7.372841 & -1.959046 & -0.762611 \\
\hline $\mathrm{C}$ & 0.758781 & 0.839527 & 0.040744 \\
\hline $\mathrm{C}$ & -0.758806 & 0.839531 & -0.040952 \\
\hline $\mathrm{C}$ & -1.565198 & 2.074219 & -0.108793 \\
\hline $\mathrm{C}$ & 1.565203 & 2.074195 & 0.108664 \\
\hline $\mathrm{C}$ & 1.413878 & 3.095504 & -0.846097 \\
\hline $\mathrm{C}$ & 2.2047 & 4.242157 & -0.787335 \\
\hline $\mathrm{C}$ & 3.140598 & 4.399311 & 0.236569 \\
\hline $\mathrm{C}$ & 3.287998 & 3.398844 & 1.200375 \\
\hline $\mathrm{C}$ & 2.512557 & 2.243408 & 1.134554 \\
\hline $\mathrm{C}$ & -1.413492 & 3.095618 & 0.845813 \\
\hline $\mathrm{C}$ & -2.204271 & 4.242309 & 0.787207 \\
\hline $\mathrm{C}$ & -3.140496 & 4.39942 & -0.236401 \\
\hline $\mathrm{C}$ & -3.288244 & 3.398885 & -1.200085 \\
\hline $\mathrm{C}$ & -2.512851 & 2.243411 & -1.134412 \\
\hline
\end{tabular}

$\begin{array}{llll}\mathrm{H} & -5.673358 & -0.362926 & 1.328941 \\ \mathrm{H} & -4.434739 & -3.617472 & -1.169471 \\ \mathrm{H} & -2.124806 & -2.696572 & -1.242062 \\ \mathrm{H} & -2.6316 & 1.465291 & -1.882431 \\ \mathrm{H} & -3.373943 & 0.57701 & 1.262888 \\ \mathrm{H} & 2.124579 & -2.696731 & 1.241648 \\ \mathrm{H} & 4.434497 & -3.617682 & 1.169241 \\ \mathrm{H} & 5.673532 & -0.362814 & -1.328545 \\ \mathrm{H} & 0.685651 & 2.983389 & -1.643217 \\ \mathrm{H} & 3.374146 & 0.577179 & -1.262665 \\ \mathrm{H} & 2.084904 & 5.015757 & -1.54064 \\ \mathrm{H} & 3.750069 & 5.297358 & 0.285327 \\ \mathrm{H} & 4.009642 & 3.516911 & 2.003864 \\ \mathrm{H} & -0.684966 & 2.983561 & 1.642668 \\ \mathrm{H} & 2.631045 & 1.465314 & 1.882656 \\ \mathrm{H} & -2.084171 & 5.015972 & 1.540398 \\ \mathrm{H} & -3.749941 & 5.29749 & -0.285045 \\ \mathrm{H} & -4.010122 & 3.516935 & -2.003366\end{array}$

$\begin{array}{llll}\mathrm{H} & -5.673358 & -0.362926 & 1.328941\end{array}$

$\begin{array}{llll}\mathrm{H} & -4.434739 & -3.617472 & -1.169471\end{array}$

$\mathrm{H}-2.124806-2.696572-1.242062$

$\begin{array}{llll}\mathrm{H} & -2.6316 & 1.465291 & -1.882431\end{array}$

$\begin{array}{lllll}\mathrm{H} & -3.373943 & 0.57701 & 1.262888\end{array}$

$\begin{array}{llll}\text { H } & 2.124579 & -2.696731 & 1.241648\end{array}$

H $5.673532 \quad-0.362814 \quad-1.328545$

$\begin{array}{lllll}\mathrm{H} & 0.685651 & 2.983389 & -1.643217\end{array}$

H $3.374146 \quad 0.577179 \quad-1.262665$

$\begin{array}{llll}\mathrm{H} & 2.084904 & 5.015757 & -1.54064\end{array}$

$\begin{array}{llll}\mathrm{H} & 3.750069 & 5.297358 & 0.285327\end{array}$

$\begin{array}{llll}\mathrm{H} & 4.009642 & 3.516911 & 2.003864\end{array}$

$\begin{array}{llll}\mathrm{H} & 2.631045 & 1.465314 & 1.882656\end{array}$

$\begin{array}{llll}\mathrm{H} & -2.084171 & 5.015972 & 1.540398\end{array}$

$\begin{array}{llll}\mathrm{H} & -4.010122 & 3.516935 & -2.003366\end{array}$ 


\begin{tabular}{|c|c|c|c|}
\hline \multicolumn{4}{|c|}{$\begin{array}{l}\text { 1c } \\
\% \mathrm{Chk}=\mathrm{COON} \\
\% \mathrm{Mem}=1 \mathrm{~GB}\end{array}$} \\
\hline \multicolumn{4}{|c|}{ B3LYP/6-31G(d) } \\
\hline \multicolumn{4}{|c|}{$\begin{array}{l}\text { COOMe_COOMe_TPCPD DFT geor } \\
\text { opt structure }\end{array}$} \\
\hline \multicolumn{4}{|c|}{41} \\
\hline & -1.248824 & -0.179084 & 0.01033 \\
\hline & -0.746133 & 1.084986 & \\
\hline & 0.770584 & 1.033477 & -0.06830 \\
\hline & 1.183405 & -0.261715 & -0 \\
\hline & -0.064004 & -1.115121 & -0. \\
\hline & -2.634627 & -0.663577 & \\
\hline & 2.531761 & -0.843573 & -0 \\
\hline & $1.6^{\prime}$ & 2.240814 & \\
\hline & -1.5 & 2.34 & \\
\hline & 1.5 & & \\
\hline & 2.3 & 4.36 & \\
\hline & 3.27 & 4.5 & \\
\hline & 3.3 & & -1 \\
\hline & 2.5 & 2.40 & 564 \\
\hline & 3.5 & -0.2 & \\
\hline & 4.8 & -0.832309 & \\
\hline & 5.1 & -1.9 & -0 \\
\hline & 4.0 & -2.5 & 468 \\
\hline & & & \\
\hline & -3.6 & 0.02 & -0. \\
\hline & -4.9 & -0.458964 & \\
\hline & -5.2 & -1.6 & \\
\hline & -4.2 & -2.3 & \\
\hline & -2.9 & -1.85 & 448 \\
\hline & -2.4 & 2.52996 & 1.1 \\
\hline & -3.1 & 3.71 & 985 \\
\hline & -2.93 & 4.727174 & 3631 \\
\hline & -2.0 & 4.556455 & 3428 \\
\hline & -1.3 & 3.381804 & 2138 \\
\hline & -0.10 & -2.33047 & 4682 \\
\hline & -6.69286 & -2.100764 & 6113 \\
\hline & -6.9 & -3.250223 & 0.4 \\
\hline & -7.573603 & -1.52335 & -0.814595 \\
\hline & 6.444329 & -2.648217 & -0.07436 \\
\hline & 6.715287 & -3.654564 & -0.70077 \\
\hline & 7.349855 & -1.991509 & 0.690505 \\
\hline
\end{tabular}

$\begin{array}{llll}\mathrm{C} & -8.247158 & -3.749974 & 0.435685 \\ \mathrm{C} & 8.662333 & -2.570674 & 0.713808 \\ \mathrm{H} & 0.771502 & 3.139524 & 1.664262 \\ \mathrm{H} & 2.234503 & 5.12818 & 1.605803 \\ \mathrm{H} & 3.909412 & 5.396236 & -0.213819 \\ \mathrm{H} & 4.113221 & 3.644205 & -1.969414 \\ \mathrm{H} & 2.668108 & 1.636522 & -1.89239 \\ \mathrm{H} & 3.376892 & 0.627439 & 1.239125 \\ \mathrm{H} & 5.629074 & -0.378672 & 1.267631 \\ \mathrm{H} & 4.2896 & -3.493328 & -1.358106 \\ \mathrm{H} & 2.015528 & -2.497666 & -1.388455 \\ \mathrm{H} & -3.375978 & 0.924459 & -1.294773 \\ \mathrm{H} & -5.701166 & 0.064097 & -1.360049 \\ \mathrm{H} & -4.555197 & -3.249299 & 1.109214 \\ \mathrm{H} & -2.234803 & -2.408355 & 1.178006 \\ \mathrm{H} & -2.599404 & 1.739737 & 1.868664 \\ \mathrm{H} & -3.900034 & 3.838642 & 2.0353 \\ \mathrm{H} & -3.571883 & 5.646262 & 0.356767 \\ \mathrm{H} & -1.916778 & 5.340924 & -1.475178 \\ \mathrm{H} & -0.596319 & 3.259139 & -1.621893 \\ \mathrm{H} & -8.237092 & -4.666554 & 1.026497 \\ \mathrm{H} & -8.547229 & -3.95966 & -0.594561 \\ \mathrm{H} & -8.942026 & -3.02298 & 0.864932 \\ \mathrm{H} & 9.253869 & -1.92836 & 1.367191 \\ \mathrm{H} & 9.090369 & -2.594968 & -0.292062 \\ \mathrm{H} & 8.626291 & -3.590367 & 1.106709\end{array}$




\begin{tabular}{|c|c|c|c|c|c|c|c|}
\hline \multicolumn{4}{|c|}{ 1d } & $\mathrm{C}$ & -10.116455 & -2.336556 & 1.689152 \\
\hline \multicolumn{4}{|c|}{ \%Chk=NHBoc_NHBoc_TPCPDHF.chk } & $\mathrm{C}$ & -10.694148 & -3.537062 & -0.45152 \\
\hline \multirow{2}{*}{\multicolumn{4}{|c|}{$\% \mathrm{Mem}=1 \mathrm{~GB}$}} & $\mathrm{C}$ & -10.587049 & -1.018416 & -0.437874 \\
\hline & & & & $\mathrm{O}$ & 7.833459 & -0.567843 & -0.753513 \\
\hline \multirow{2}{*}{\multicolumn{4}{|c|}{ \# B3LYP/6-31G(d) }} & $\mathrm{C}$ & 10.015939 & -2.308356 & -0.159893 \\
\hline & & & & $\mathrm{C}$ & 10.693956 & -3.537464 & 0.45134 \\
\hline \multicolumn{4}{|c|}{ NHBoc_NHBoc_TPCPD geom opt } & $\mathrm{C}$ & 10.586694 & -1.018812 & 0.439893 \\
\hline \multicolumn{4}{|c|}{$01--50$} & $\mathrm{C}$ & 10.117256 & -2.335096 & -1.688545 \\
\hline $\mathrm{C}$ & -1.210632 & 0.199715 & -0.15578 & $\mathrm{H}$ & -2.48692 & 2.118176 & -2.068538 \\
\hline & -0.752531 & 1.480117 & -0.101172 & $\mathrm{H}$ & -3.843035 & 4.177499 & -2.288888 \\
\hline C & 0.752529 & 1.480063 & 0.100952 & $\mathrm{H}$ & -3.702327 & 5.955564 & -0.553135 \\
\hline 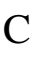 & 1.21057 & 0.199629 & 0.155328 & $\mathrm{H}$ & -2.17936 & 5.659613 & 1.391725 \\
\hline $\mathrm{C}$ & -0.00005 & -0.694009 & -0.000319 & $\mathrm{H}$ & -0.805067 & 3.617212 & 1.593667 \\
\hline $\mathrm{O}$ & -0.000076 & -1.911786 & -0.000454 & $\mathrm{H}$ & -2.029626 & -2.072945 & -1.386233 \\
\hline $\mathrm{C}$ & -2.57429 & -0.33733 & -0.220779 & $\mathrm{H}$ & -4.301056 & -2.994485 & -1.494109 \\
\hline $\mathrm{C}$ & 2.574197 & -0.337511 & 0.220272 & $\mathrm{H}$ & -5.762628 & 0.285718 & 0.872385 \\
\hline $\mathrm{C}$ & 1.552465 & 2.71675 & 0.220909 & $\mathrm{H}$ & -3.487103 & 1.211809 & 173 \\
\hline $\mathrm{C}$ & -1.552396 & 2.716869 & -0.220929 & $\mathrm{H}$ & 3.4 & 1.211855 & 257 \\
\hline $\mathrm{C}$ & -2.419189 & 2.895992 & -1.313982 & $\mathrm{H}$ & 5.762644 & 0.285617 & -0.872526 \\
\hline $\mathrm{C}$ & -3.182651 & 4.055113 & -1.434556 & $\mathrm{H}$ & 4.300722 & -2.995073 & 1.493074 \\
\hline $\mathrm{C}$ & -3.102844 & 5.053888 & -0.461036 & $\mathrm{H}$ & 2.029354 & -2.073371 & 1.385279 \\
\hline $\mathrm{C}$ & -2.247444 & 4.888168 & 0.629382 & $\mathrm{H}$ & 2.486882 & 2.11776 & 2.068477 \\
\hline $\mathrm{C}$ & -1.471329 & 3.735378 & 0.744843 & $\mathrm{H}$ & 3.843121 & 4.176969 & 2.289137 \\
\hline $\mathrm{C}$ & -2.839446 & -1.541963 & -0.900829 & $\mathrm{H}$ & & & \\
\hline $\mathrm{C}$ & -4.124176 & -2.065346 & -0.955639 & $\mathrm{H}$ & 2.179668 & 5.659664 & 1342 \\
\hline $\mathrm{C}$ & -5.195269 & -1.4131 & -0.325434 & $\mathrm{H}$ & 0.805249 & 3.617372 & -1.593592 \\
\hline $\mathrm{C}$ & -4.94879 & -0.218389 & $0.3^{\circ}$ & $\mathrm{H}$ & -6.515988 & -2.875987 & -0.944284 \\
\hline $\mathrm{C}$ & -3.65861 & 0.297117 & 0.41 & $\mathrm{H}$ & 6.515637 & -2.876736 & 0.943172 \\
\hline $\mathrm{C}$ & 3.658593 & 0.297022 & -0.415036 & $\mathrm{H}$ & -11.170889 & -2.326314 & 1.98749 \\
\hline $\mathrm{C}$ & 4.948741 & -0.218571 & -0.370408 & $\mathrm{H}$ & -9.617824 & -1.473269 & 2.132261 \\
\hline $\mathrm{C}$ & 5.195101 & -1.413458 & 0.324841 & $\mathrm{H}$ & -9.660442 & -3.251232 & 2.083731 \\
\hline $\mathrm{C}$ & 4.12393 & -2.065795 & 0.954817 & $\mathrm{H}$ & -11.7634 & -3.531908 & -0.215773 \\
\hline $\mathrm{C}$ & 2.839235 & -1.542322 & 0.90005 & $\mathrm{H}$ & -10.256405 & -4.459026 & -0.055166 \\
\hline $\mathrm{C}$ & 2.419227 & 2.895676 & 1.314018 & $\mathrm{H}$ & -10.579586 & $5-3.54018$ & -1.540421 \\
\hline $\mathrm{C}$ & 3.182758 & 4.054735 & 1.434768 & $\mathrm{H}$ & -11.659949 & -0.959003 & -0.222993 \\
\hline $\mathrm{C}$ & 3.103051 & 5.053638 & 0.461372 & $\mathrm{H}$ & -10.457983 & $3-1.013737$ & -1.525845 \\
\hline $\mathrm{C}$ & 2.247677 & 4.888115 & -0.629098 & $\mathrm{H}$ & -10.095988 & -0.138464 & -0.019643 \\
\hline $\mathrm{C}$ & 1.471493 & 3.73539 & -0.744733 & $\mathrm{H}$ & 11.763325 & -3.532028 & 0.216128 \\
\hline $\mathrm{N}$ & -6.464296 & -2.009105 & -0.426705 & $\mathrm{H}$ & 10.256474 & -4.459105 & 0.053945 \\
\hline $\mathrm{N}$ & 6.464075 & -2.009608 & 0.425993 & $\mathrm{H}$ & 10.578853 & -3.541559 & 1.54018 \\
\hline $\mathrm{C}$ & 7.662938 & -1.578638 & -0.095907 & $\mathrm{H}$ & 11.65967 & -0.959059 & 0.225491 \\
\hline $\mathrm{C}$ & -7.662974 & -1.578545 & 0.095969 & $\mathrm{H}$ & 10.457195 & -1.015164 & 1.527817 \\
\hline $\mathrm{O}$ & -8.613535 & -2.480628 & -0.25569 & $\mathrm{H}$ & 10.095698 & -0.138527 & 0.022284 \\
\hline $\mathrm{O}$ & 8.613383 & -2.480975 & 0.255435 & $\mathrm{H}$ & 11.171838 & -2.324595 & -1.986359 \\
\hline $\mathrm{O}$ & -7.833266 & -0.568213 & 0.754345 & $\mathrm{H}$ & 9.618842 & -1.471421 & -2.131137 \\
\hline $\mathrm{C}$ & -10.015907 & $7-2.308457$ & 0.160472 & $\mathrm{H}$ & 9.661437 & -3.249421 & -2.08416 \\
\hline
\end{tabular}




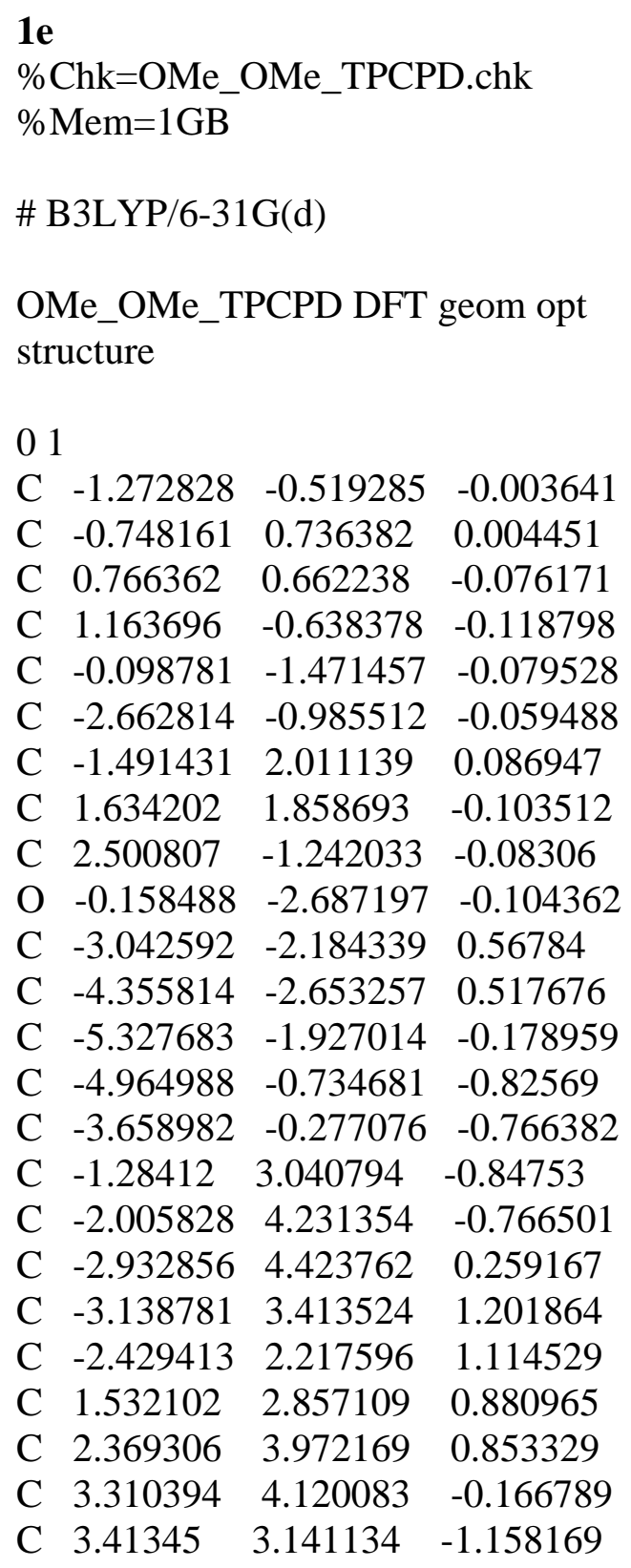
$\begin{array}{llll}\text { C } & 2.587369 & 2.019584 & -1.12513\end{array}$
$\begin{array}{llll}\text { C } & 2.766581 & -2.446349 & -0.770814\end{array}$
$\begin{array}{llll}\text { C } & 4.023736 & -3.03094 & -0.738096\end{array}$
$\begin{array}{llll}\text { C } & 5.063245 & -2.43803 & -0.007146\end{array}$
C $4.818768 \quad-1.251691 \quad 0.696582$
$\begin{array}{llll}\text { C } & 3.552123 & -0.671879 & 0.654172\end{array}$
$\begin{array}{llll}\text { O } & -6.638144 & -2.288573 & -0.293848\end{array}$
$\begin{array}{lllll}\text { O } & 6.26184 & -3.089319 & -0.040194\end{array}$
$\begin{array}{llll}\text { C } & -7.058949 & -3.493689 & 0.325686\end{array}$
$\begin{array}{llll}\text { C } & 7.348057 & -2.534769 & 0.683975\end{array}$
H $\quad-2.298996 \quad-2.76466 \quad 1.100816$
$\begin{array}{llll}\mathrm{H} & -4.604645 & -3.58031 & 1.021484\end{array}$
H $\quad-5.726172 \quad-0.191154-1.376878$
$\begin{array}{llll}\mathrm{H} & -3.39649 & 0.637248 & -1.287587\end{array}$
$\mathrm{H} \quad-0.561878 \quad 2.902016 \quad-1.646113$
$\begin{array}{llll}\mathrm{H} & -1.838755 & 5.011345 & -1.504555\end{array}$
$\mathrm{H} \quad-3.489434 \quad 5.354769 \quad 0.325353$
$\begin{array}{llll}\mathrm{H} & -3.85509 & 3.556057 & 2.006666\end{array}$
$\begin{array}{llll}\mathrm{H} & -2.595546 & 1.431394 & 1.844811\end{array}$
$\begin{array}{llll}\mathrm{H} & 0.799209 & 2.753564 & 1.675188\end{array}$
$\begin{array}{llll}\mathrm{H} & 2.281308 & 4.728754 & 1.628507\end{array}$
H $3.956944 \quad 4.993216 \quad-0.191623$
H $4.1392893 .250546 \quad-1.959648$
$\begin{array}{llll}\mathrm{H} & 2.672713 & 1.257292 & -1.893668\end{array}$
$\begin{array}{llll}\mathrm{H} & 1.973363 & -2.925027 & -1.332473\end{array}$
H $4.226882 \quad-3.952988-1.273941$
H $5.597253 \quad-0.77871 \quad 1.284486$
$\begin{array}{llll}\mathrm{H} & 3.376285 & 0.23741 & 1.21909\end{array}$
$\begin{array}{llll}\mathrm{H} & -8.124246 & -3.586771 & 0.106448\end{array}$
$\begin{array}{llll}\mathrm{H} & -6.914306 & -3.460335 & 1.413688\end{array}$
H $-6.527247-4.363936-0.081085$
H $8.193159 \quad-3.205042 \quad 0.516251$
$\begin{array}{llll}\text { H } & 7.133329 & -2.48475 & 1.75971\end{array}$
H $7.604339 \quad-1.530317 \quad 0.321638$ 


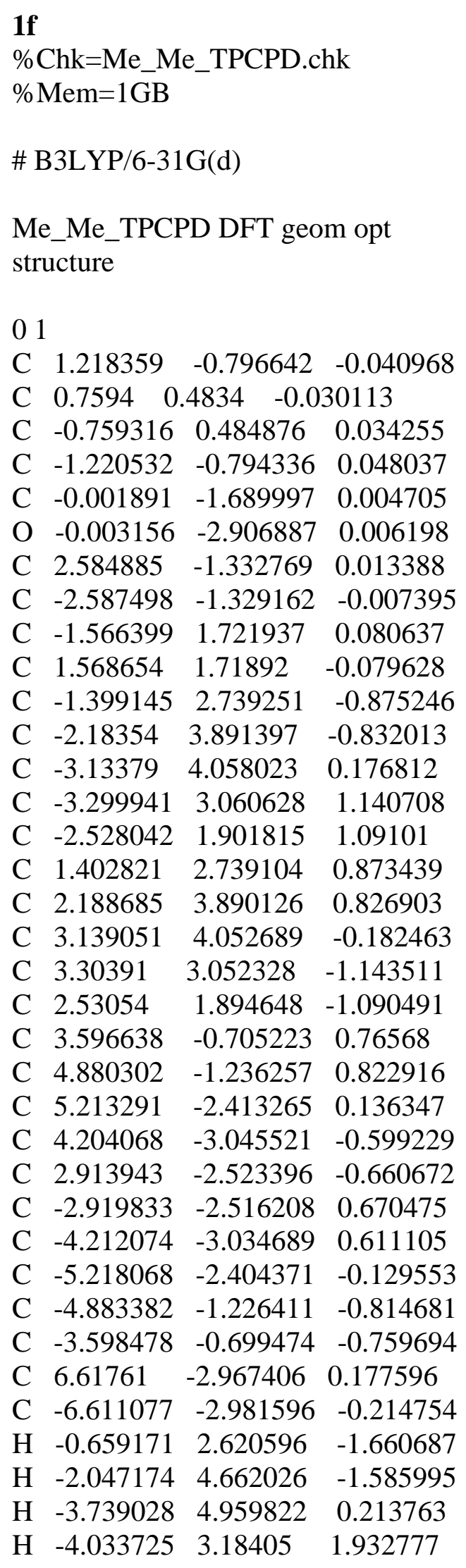

$$
\begin{array}{llll}
\mathrm{H} & -2.663196 & 1.124921 & 1.837399 \\
\mathrm{H} & 0.662682 & 2.623672 & 1.659197 \\
\mathrm{H} & 2.053318 & 4.663089 & 1.578669 \\
\mathrm{H} & 3.745384 & 4.953641 & -0.222077 \\
\mathrm{H} & 4.03777 & 3.172622 & -1.935993 \\
\mathrm{H} & 2.664545 & 1.115523 & -1.834752 \\
\mathrm{H} & 3.366438 & 0.196451 & 1.323556 \\
\mathrm{H} & 5.637698 & -0.733006 & 1.420412 \\
\mathrm{H} & 4.429173 & -3.966303 & -1.133042 \\
\mathrm{H} & 2.15135 & -3.043933 & -1.227928 \\
\mathrm{H} & -2.160533 & -3.033684 & 1.244921 \\
\mathrm{H} & -4.441478 & -3.949888 & 1.152538 \\
\mathrm{H} & -5.641511 & -0.717905 & -1.406941 \\
\mathrm{H} & -3.367807 & 0.204476 & -1.313618 \\
\mathrm{H} & 6.632708 & -4.038482 & -0.049476 \\
\mathrm{H} & 7.077761 & -2.823264 & 1.161578 \\
\mathrm{H} & 7.265023 & -2.469059 & -0.556928 \\
\mathrm{H} & -7.375937 & -2.202745 & -0.113342 \\
\mathrm{H} & -6.783616 & -3.728526 & 0.566823 \\
\mathrm{H} & -6.780347 & -3.473509 & -1.182042
\end{array}
$$




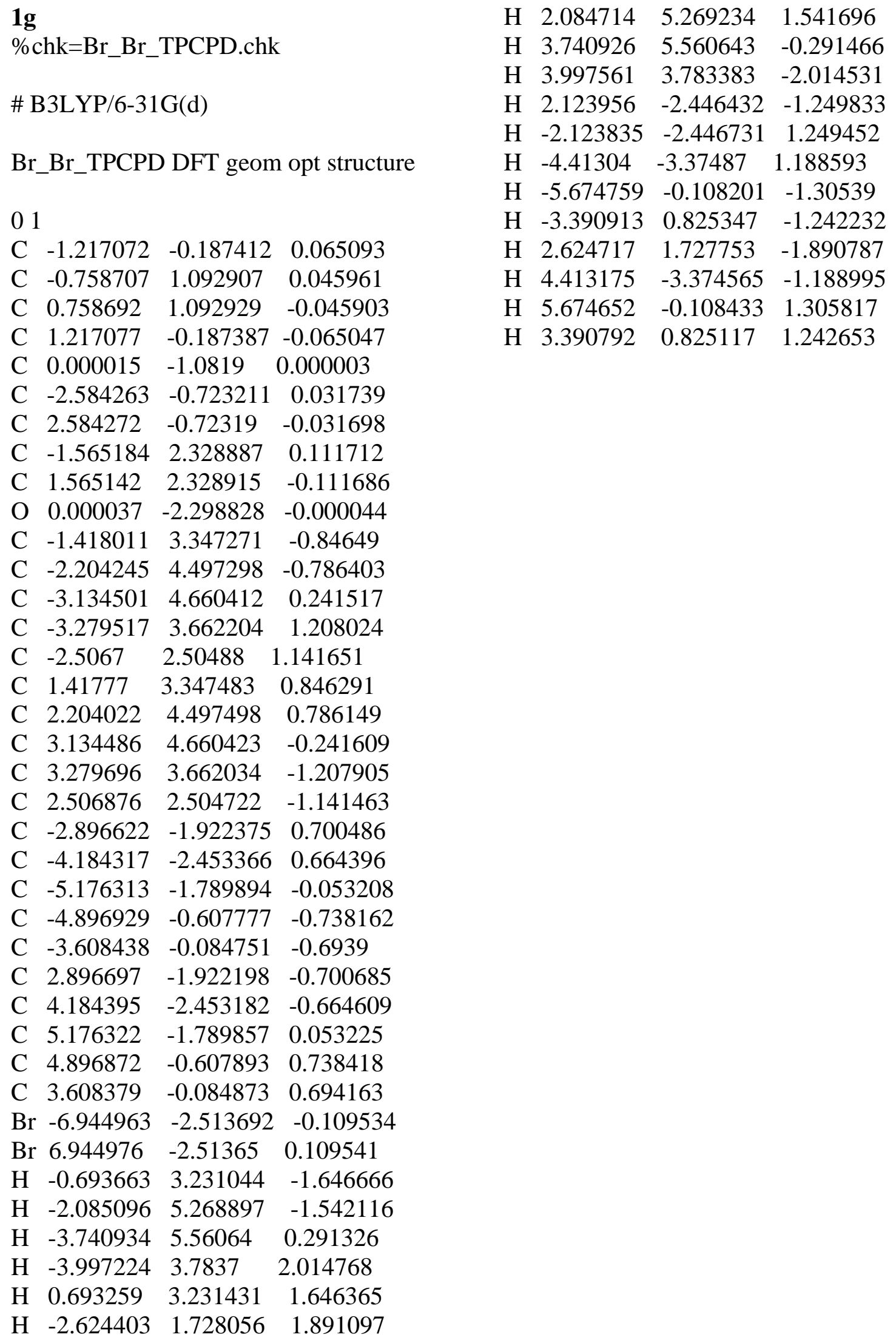




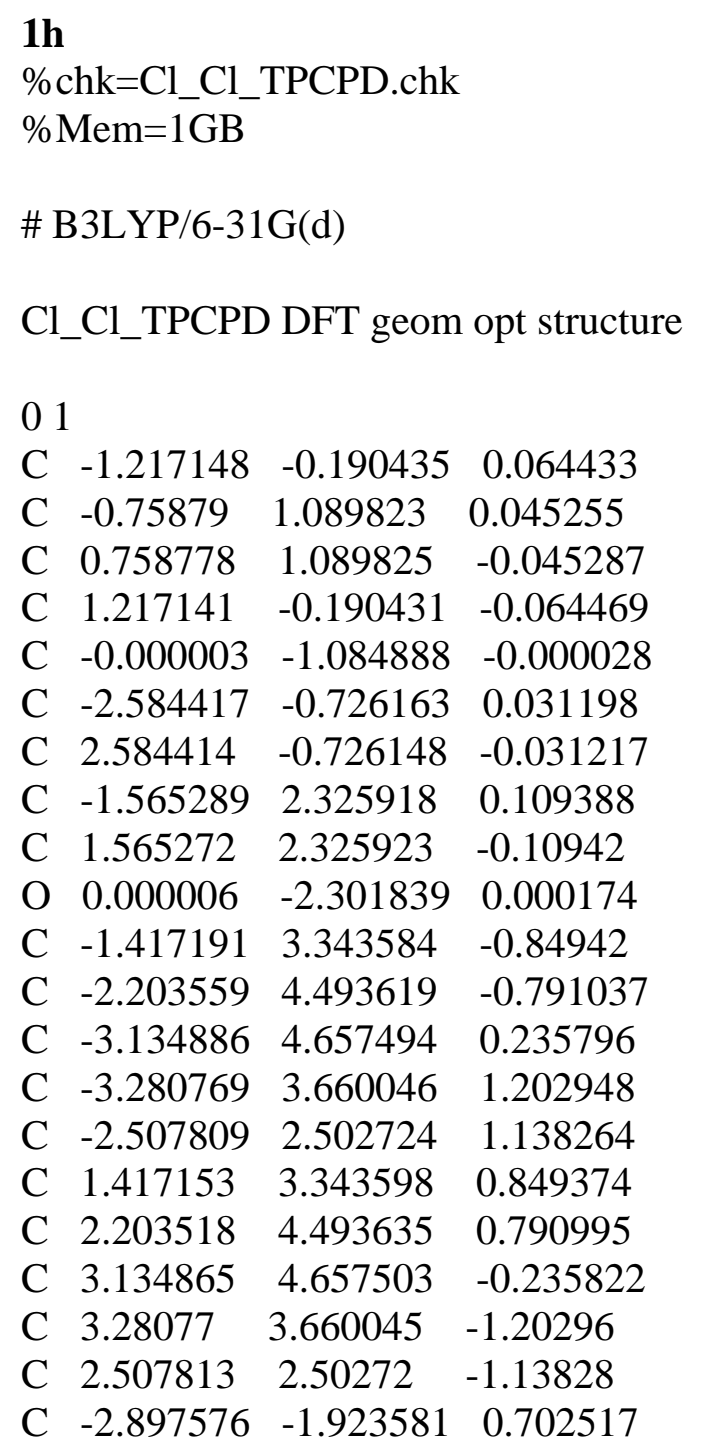
$\begin{array}{llll}\text { C } & -4.185544 & -2.453461 & 0.669112\end{array}$
$\begin{array}{llll}\text { C } & -5.178455 & -1.790297 & -0.048848\end{array}$
$\begin{array}{llll}\text { C } & -4.897472 & -0.610063 & -0.737754\end{array}$
$\begin{array}{llll}\text { C } & -3.608361 & -0.088922 & -0.695633\end{array}$
$\begin{array}{llll}\text { C } & 2.897598 & -1.923559 & -0.702539\end{array}$
$\begin{array}{llll}\text { C } & 4.185572 & -2.453424 & -0.669115\end{array}$
$\begin{array}{llll}\text { C } & 5.178464 & -1.790253 & 0.048864\end{array}$
$\begin{array}{llll}\text { C } & 4.897457 & -0.610025 & 0.737769\end{array}$
$\begin{array}{llll}\text { C } 3.60834 & -0.088899 & 0.695631\end{array}$
$\begin{array}{llll}\mathrm{Cl} & -6.807198 & -2.452445 & -0.094435\end{array}$
Cl $6.807214 \quad-2.452382 \quad 0.094473$
$\begin{array}{lllll}\mathrm{H} & -0.692012 & 3.226816 & -1.648778\end{array}$
$\begin{array}{llll}\mathrm{H} & -2.083628 & 5.264646 & -1.547213\end{array}$
$\begin{array}{llll}\mathrm{H} & -3.741418 & 5.557727 & 0.284272\end{array}$
$\begin{array}{llll}\mathrm{H} & -3.999277 & 3.782151 & 2.008893\end{array}$
$\mathrm{H} \quad 0.69196 \quad 3.226835 \quad 1.64872$


$\begin{array}{llll}\mathrm{H} & 2.08357 & 5.264668 & 1.547161\end{array}$
H $3.741395 \quad 5.557737 \quad-0.284295$
H $3.999294 \quad 3.782143 \quad-2.008892$
H $2.124736 \quad-2.44722 \quad-1.25199$

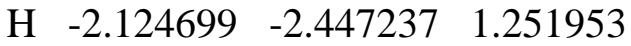
H $-4.416154 \quad-3.373525 \quad 1.195526$
$\begin{array}{llll}\mathrm{H} & -5.676048 & -0.11203 & -1.305795\end{array}$

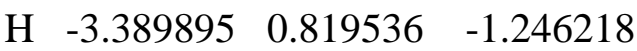
$\begin{array}{llll}\mathrm{H} & 2.626297 & 1.726424 & -1.888198\end{array}$
H $4.416201 \quad-3.373482 \quad-1.195531$
H $5.676018 \quad-0.1119841 .305825$
$\begin{array}{llll}\text { H } & 3.389856 & 0.819557 & 1.246214\end{array}$ 


\section{Calculation of Potential Energy as a function of Phenyl-CP ring dihedrals}

Optimization of the unsubstituted TPCPD was also conducted at the same level of theory while confining the structure to the $\mathrm{C} 2$ point group. Many variables were introduced in order to avoid converging on a local minimum that was not representative of the natural structure. The resulting structure was slightly higher in energy $(-1192.329 \mathrm{H}$ vs $-1192.339 \mathrm{H})$ than the unenforced optimization, but by adjusting the Z-matrix further one could, in principle, develop a C2 structure that converges at a point within the margin of error of the method.

$\% \mathrm{Chk}=\mathrm{C} 2 \mathrm{Opt} 2 . \mathrm{chk}$

$\% \mathrm{Mem}=1 \mathrm{~GB}$

\# B3LYP/6-31G(d) Opt=Z-matrix

C2 Optimization with many floating variables

01

$\mathrm{XXX}$

$\mathrm{XX}$ XXX 1.0

X XX 1.0 XXX 90.0

C1 X cx XX 90.0 XXX 0.0

C2 X cXX XX 90.0 XXX d001

C3 X cXXX XX 90.0 XXX d002

C4 X cXXX XX 90.0 XXX -d002

C5 X cXX XX 90.0 XXX -d001

O X ox XX 90.0 XXX 0.0

XXXX X 1.0 XXX 135.0 XX 180.0

C6 X ccr1 XX 90.0 XXX d001

C7 C6 ri1 X pha1 XX d004

C8 C6 ri1 X pha1 XXXX d004

C9 C7 ri3 C6 pha3 $\mathrm{X}$ d006

C10 C8 ri3 C6 pha3 X d006

C11 X rL1 XX 90.0 XXX d008

C12 X ccr1 XX 90.0 XXX -d001

C13 C12 ri1 X pha1 XXXX d004

C14 C12 ri1 $X$ pha1 XX d004

C15 C13 ri3 C12 pha3 X d006

C16 C14 ri3 C12 pha3 X d006

C17 X rL1 XX 90.0 XXX -d008

C18 X ccr2 XX 90.0 XXX d002

C19 C18 ri1 X pha1 XX d004

C20 C18 ri1 X pha1 XXXX d004

C21 C19 ri3 C18 pha3 X d006

C22 C20 ri3 C18 pha3 X d006

C23 X rL2 XX 90.0 XXX d018 
C24 X ccr2 XX 90.0 XXX -d002

C25 C24 ri1 X pha1 XXXX d004

C26 C24 ri1 X pha1 XX d004

C27 C25 ri3 C24 pha3 X d006

C28 C26 ri3 C24 pha3 X d006

C29 X rL2 XX 90.0 XXX -d018

H1 C7 ch1 C6 cha1 C8 dh1

$\mathrm{H} 2 \mathrm{C} 8$ ch $1 \mathrm{C} 6$ cha1 $\mathrm{C} 7 \mathrm{dh} 1$

H3 C9 ch1 C7 cha3 C6 dh1

H4 C10 ch1 C8 cha3 C6 dh1

H5 X hL XX 90.0 XXX dh01

H6 C13 ch1 C12 cha1 C14 dh1

H7 C14 ch1 C12 cha1 C13 dh1

H8 C15 ch1 C13 cha3 C12 dh1

H9 C16 ch1 C14 cha3 C12 dh1

H10 X hL XX 90.0 XXX -dh01

H11 C19 ch2 $\mathrm{C} 18$ cha1 C23 dh1

H12 C20 ch2 $\mathrm{C} 18$ cha1 $\mathrm{C} 23 \mathrm{dh} 1$

H13 C21 ch2 C19 cha3 C18 dh1

H14 C22 ch2 220 cha3 C18 dh1

H15 X hL2 XX 90.0 XXX dh02

H16 C25 ch2 C24 cha1 C29 dh1

H17 C26 ch2 C24 cha1 C29 dh1

H18 C27 ch2 C25 cha3 C24 dh1

H19 C28 ch2 C26 cha3 C24 dh1

H20 X hL2 XX 90.0 XXX -dh02

$$
\begin{aligned}
& \mathrm{hL}=6.5985 \\
& \mathrm{hL} 2=6.5736 \\
& \text { dh01 }=73.716 \\
& \text { dh02 }=142.4296 \\
& \text { cha } 1=119.4481 \\
& \text { cha3 }=119.7677 \\
& \text { dh } 1=180.1678 \\
& \text { cx }=1.2385 \\
& \text { cXX }=1.2389 \\
& \text { cXXX }=1.2109 \\
& \text { ccr1 }=2.7076 \\
& \text { ccr2 }=2.6883 \\
& \text { ox }=2.4544 \\
& \text { ri1 } 1=1.406 \\
& \text { ri3 }=1.3926 \\
& \text { pha1 } 1=120.6567 \\
& \text { pha3 }=120.5721 \\
& \text { d001 }=73.7411 \\
& \text { d002 }=142.3456
\end{aligned}
$$




$$
\begin{aligned}
& \mathrm{d} 004=139.5639 \\
& \mathrm{~d} 006=180.1952 \\
& \mathrm{~d} 008=73.7223 \\
& \mathrm{~d} 018=142.4433 \\
& \mathrm{rL} 1=5.5146 \\
& \mathrm{rL} 2=5.4877 \\
& \operatorname{ch} 1=1.0861 \\
& \operatorname{ch} 2=1.086
\end{aligned}
$$

Using values obtained from the optimization of the prior Z-matrix a $\mathrm{C} 2 \mathrm{v}$ single point calculation was performed. This structure was referred to as $\mathbf{1 a}\left(\mathbf{C}_{\mathbf{2 v}}\right)$ in the text and had all four phenyl rings orthogonal to the CPD ring. A dihedral drive was performed on this structure by varying angle ? (d004 in Z-matrix) by 10 degree increments. These structures can be thought of as platonic solids of the natural structure and only serve as a tool to see how the conjugation from the pendant phenyl rings perturbs the resulting molecular orbitals.

\%chk=PES_0.chk

$\% \mathrm{Mem}=1 \mathrm{~GB}$

\# HF/6-31G(d)

PES_0 C2v point group: variables fixed for dihedral drive

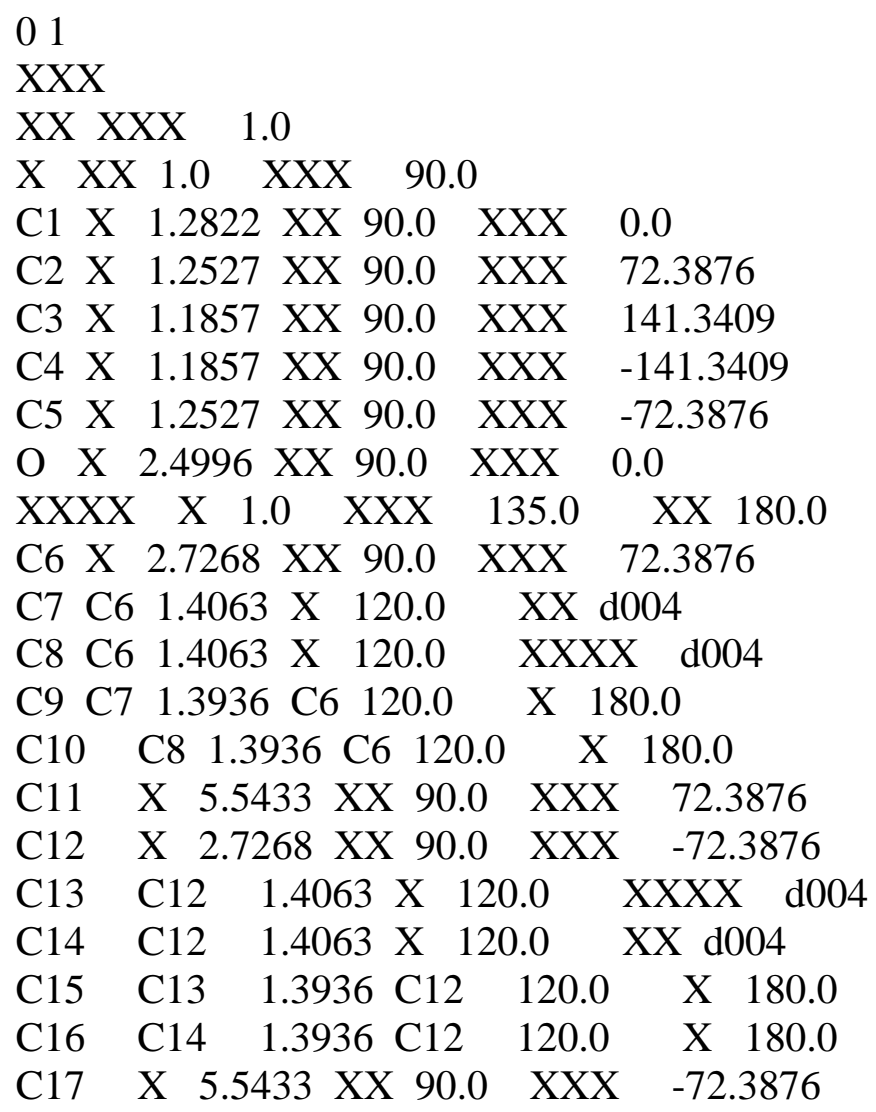




\begin{tabular}{|c|c|c|c|c|}
\hline 18 & X 2.6647 XX 90.0 & $\mathrm{XX}$ & & 409 \\
\hline 19 & $\mathrm{C} 18 \quad 1.4063 \times 12$ & & $\mathrm{XX} \mathrm{d} 0$ & \\
\hline 20 & $1.4063 \times 12$ & & XXXX & d004 \\
\hline 21 & $1.3936 \mathrm{C} 18$ & 120.0 & & 180.0 \\
\hline $\mathrm{C} 22$ & $1.3936 \mathrm{C} 18$ & 120.0 & & 180.0 \\
\hline 23 & X 5.4806 XX 90.0 & XXX & 141.3 & 3409 \\
\hline 24 & X 2.6647 XX 90.0 & XXX & -141 & 1.3409 \\
\hline 25 & $1.4063 \times 12$ & & XXXX & d004 \\
\hline $\mathrm{C} 26$ & $1.4063 \times 12$ & & $\mathrm{XX} \mathrm{d} 0$ & 004 \\
\hline 27 & $1.3936 \mathrm{C} 24$ & 120.0 & $\mathrm{X}$ & 180.0 \\
\hline 28 & $1.3936 \mathrm{C} 24$ & 120.0 & & 180.0 \\
\hline 29 & X 5.4806 XX 90.0 & XXX & -141 & 1.3409 \\
\hline $\mathrm{H} 1 \mathrm{C}^{\prime}$ & $\begin{array}{llll}7 & 1.0862 & \text { C6 } & 120.0\end{array}$ & & 80.0 & \\
\hline $\mathrm{H} 2 \mathrm{C}$ & $\begin{array}{llll}8 & 1.0862 & \text { C6 } & 120.0\end{array}$ & C7 18 & 80.0 & \\
\hline $\mathrm{H} 3 \mathrm{C}$ & $\begin{array}{llll}9 & 1.0862 & \text { C7 } & 120.0\end{array}$ & C6 18 & 80.0 & \\
\hline $\mathrm{H} 4 \mathrm{C}$ & $\begin{array}{lll}1.0862 & C 8 & 120.0\end{array}$ & C6 & 6180.0 & \\
\hline H5 X & 6.6301 XX $90.0 \quad$ X & $\mathrm{XX}$ & 72.3876 & \\
\hline $\mathrm{H} 6 \mathrm{Cl}$ & $1.0862 \mathrm{C} 12$ & 0.0 & & 180.0 \\
\hline $7 \mathrm{C}$ & $1.0862 \mathrm{C} 12$ & 0.0 & $\mathrm{C} 13$ & 180.0 \\
\hline $\mathrm{H} 8 \mathrm{Cl}$ & $1.0862 \mathrm{C} 13$ & 0.0 & $\mathrm{C} 12$ & 180.0 \\
\hline $\mathrm{H} 9 \mathrm{C}$ & $1.0862 \mathrm{C} 14$ & 0.0 & $\mathrm{C} 12$ & 180.0 \\
\hline $\mathrm{H} 10$ & $\begin{array}{llll}X & 6.6301 & \text { XX } & 90.0\end{array}$ & XXX & -72.3 & 3876 \\
\hline H11 & $1.0861 \mathrm{C} 18$ & 120.0 & $\mathrm{C} 23$ & 180.0 \\
\hline 12 & $1.0861 \mathrm{C} 18$ & 120.0 & $\mathrm{C} 23$ & 180.0 \\
\hline 13 & $1.0861 \mathrm{C} 19$ & 120.0 & $\mathrm{C} 18$ & 180.0 \\
\hline $\mathrm{I} 14$ & $1.0861 \mathrm{C} 20$ & 120.0 & $\mathrm{C} 18$ & 180.0 \\
\hline H15 & $\begin{array}{lll}X & 6.5673 \text { XX } 90.0\end{array}$ & XXX & 141. & 3409 \\
\hline H16 & $1.0861 \mathrm{C} 24$ & 120.0 & $\mathrm{C} 29$ & 180.0 \\
\hline 17 & $1.0861 \mathrm{C} 24$ & 120.0 & $\mathrm{C} 29$ & 180.0 \\
\hline 18 & $1.0861 \mathrm{C} 25$ & 120.0 & $\mathrm{C} 24$ & 180.0 \\
\hline $\mathrm{I} 19$ & $1.0861 \mathrm{C} 26$ & 120.0 & $\mathrm{C} 24$ & 180.0 \\
\hline & X 6.5673 XX 90.0 & XXX & -141 & 1.3409 \\
\hline
\end{tabular}

By substituting 90.0 for d004=0.0 in the matrix above one can obtain the structure where all rings occupy the same plane. Due to the Pauli repulsion of neighboring atoms in the molecule this structure is $7850 \mathrm{kcal} / \mathrm{mol}$ higher in energy than the fully optimized structure. 


\section{Computations of excited States of TACPD model compounds}

Prediction of Excited States for 2:

The following Gaussian input file gave the output below for the single excitations within the CIS method.

\section{Input File:}

$\% \mathrm{Chk}=\mathrm{CPDC} 2 \mathrm{vCIS} 30 . \mathrm{chk}$

\# RCIS=(NStates=30, FC, Direct)/6-31G(d)

CPDC2vCIS30

01

$\mathrm{X} 3$

$\mathrm{X} 2 \mathrm{X} 31.0$

$\mathrm{X} \times 21.0 \times 390.0$

C1 X 1.50270425 X290.0 X3 0.0

C2 X 1.34303547 X2 90.0 X3 63.84677685

C3 X 1.00633596 X2 90.0 X3 131.67443774

C4 X 1.00633596 X2 90.0 X3 -131.67443774

C5 X 1.34303547 X2 90.0 X3 -63.84677685

O X 2.71716821 X2 90.0 X3 0.0

H1 X 2.42082672 X2 90.0 X3 66.84685352

H2 X 2.07270798 X2 90.0 X3 139.52404558

H3 X 2.07270798 X2 90.0 X3 -139.52404558

H4 X 2.42082672 X2 90.0 X3 -66.84685352

\section{Output File:}

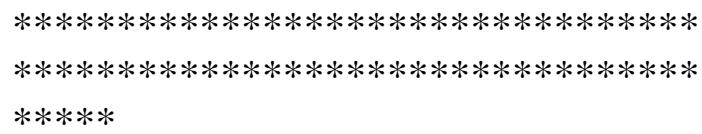

Excited states from <AA,BB:AA,BB> singles matrix:

$* * * * * * * * * * * * * * * * * * * * * * * * * * * * * * * * *$

$* * * * * * * * * * * * * * * * * * * * * * * * * * * * * * * * * *$

$* * * * *$

Ground to excited state Transition electric dipole moments (Au):

\begin{tabular}{ccccc} 
state & $\mathrm{X}$ & $\mathrm{Y}$ & $\mathrm{Z}$ & \multicolumn{2}{c}{ Osc. } \\
1 & .0000 & .0000 & .0000 & .0000 \\
2 & .0000 & .5410 & .0000 & .0289
\end{tabular}

$\begin{array}{lllll}3 & .0000 & .0000 & -1.7422 & .5730\end{array}$

$\begin{array}{lllll}4 & -.0735 & .0000 & .0000 & .0011\end{array}$

$\begin{array}{llllll}5 & .0000 & .0000 & .0000 & .0000\end{array}$

$\begin{array}{llllll}6 & -.0770 & .0000 & .0000 & .0013\end{array}$

$\begin{array}{llllll}7 & .0000 & .0000 & .0000 & .0000\end{array}$

$\begin{array}{llllll}8 & .0000 & .0000 & .0000 & .0000\end{array}$

$\begin{array}{llllll}9 & .0000 & .0000 & 1.5920 & .5939\end{array}$

$\begin{array}{lllll}10 & .0000 & .0000 & .3414 & .0288\end{array}$

$\begin{array}{lllll}11 & .0000 & .7905 & .0000 & .1591\end{array}$

$\begin{array}{llllll}12 & .0179 & .0000 & .0000 & .0001\end{array}$

$\begin{array}{llllll}13 & -.0183 & .0000 & .0000 & .0001\end{array}$

$\begin{array}{llllll}14 & .0000 & .0000 & .0000 & .0000\end{array}$

$\begin{array}{llllll}15 & .0055 & .0000 & .0000 & .0000\end{array}$

$\begin{array}{lllll}16 & .0000 & .0000 & .0000 & .0000\end{array}$

$\begin{array}{llllll}17 & -.0768 & .0000 & .0000 & .0017\end{array}$ 


$$
\begin{array}{lrrrr}
18 & .0000 & .4362 & .0000 & .0558 \\
19 & .0000 & .0000 & .0000 & .0000 \\
20 & .0000 & .0000 & .0000 & .0000 \\
21 & -.0088 & .0000 & .0000 & .0000 \\
22 & .0000 & -.4806 & .0000 & .0713 \\
23 & .0000 & .0000 & -.0300 & .0003 \\
24 & .0464 & .0000 & .0000 & .0007 \\
25 & .0000 & -1.2406 & .0000 & .5223 \\
26 & .0505 & .0000 & .0000 & .0009 \\
27 & .0000 & .0000 & .0000 & .0000 \\
28 & .0000 & .0000 & -.5849 & .1174 \\
29 & .0000 & .6647 & .0000 & .1554 \\
30 & .0000 & .0000 & .9586 & .3280
\end{array}
$$

Ground to excited state transition velocity dipole Moments ( $\mathrm{Au})$ :

$$
\begin{array}{ccccc}
\text { state } & \mathrm{X} & \mathrm{Y} & \mathrm{Z} & \multicolumn{2}{l}{\text { Osc. }} \\
1 & .0000 & .0000 & .0000 & .0000 \\
2 & .0000 & .0129 & .0000 & .0008 \\
3 & .0000 & .0000 & .1157 & .0315 \\
4 & .0292 & .0000 & .0000 & .0019 \\
5 & .0000 & .0000 & .0000 & .0000 \\
6 & .0246 & .0000 & .0000 & .0013 \\
7 & .0000 & .0000 & .0000 & .0000 \\
8 & .0000 & .0000 & .0000 & .0000 \\
9 & .0000 & .0000 & -.2042 & .0791 \\
10 & .0000 & .0000 & -.0353 & .0022 \\
11 & .0000 & -.1170 & .0000 & .0239 \\
12 & .0397 & .0000 & .0000 & .0027 \\
13 & -.0047 & .0000 & .0000 & .0000 \\
14 & .0000 & .0000 & .0000 & .0000 \\
15 & .0496 & .0000 & .0000 & .0040 \\
16 & .0000 & .0000 & .0000 & .0000 \\
17 & .0253 & .0000 & .0000 & .0010 \\
18 & .0000 & -.0762 & .0000 & .0088 \\
19 & .0000 & .0000 & .0000 & .0000 \\
20 & .0000 & .0000 & .0000 & .0000 \\
21 & -.0442 & .0000 & .0000 & .0029 \\
22 & .0000 & .1531 & .0000 & .0337 \\
23 & .0000 & .0000 & .0102 & .0001 \\
24 & -.0573 & .0000 & .0000 & .0044 \\
25 & .0000 & .5726 & .0000 & .4293 \\
26 & -.0273 & .0000 & .0000 & .0010 \\
27 & .0000 & .0000 & .0000 & .0000 \\
28 & .0000 & .0000 & .2472 & .0791 \\
29 & .0000 & -.2475 & .0000 & .0774 \\
30 & .0000 & .0000 & -.5130 & .3276
\end{array}
$$

Ground to excited state transition magnetic dipole Moments ( $\mathrm{Au}$ ):

$$
\begin{aligned}
& \text { state } \mathrm{X} \quad \mathrm{Y} \quad \mathrm{Z} \\
& \begin{array}{lllll}
1 & .0000 & .0000 & -.7331
\end{array} \\
& \begin{array}{lllll}
2 & -1.2497 & .0000 & .0000
\end{array} \\
& \begin{array}{llll}
3 & .0000 & .0000 & .0000
\end{array} \\
& \begin{array}{llll}
4 & .0000 & .5806 & .0000
\end{array} \\
& \begin{array}{lllll}
5 & .0000 & .0000 & -.8129
\end{array} \\
& \begin{array}{llll}
6 & .0000 & -.2609 & .0000
\end{array} \\
& \begin{array}{lllll}
7 & .0000 & .0000 & .8662
\end{array} \\
& \begin{array}{lllll}
8 & .0000 & .0000 & -.1026
\end{array} \\
& \begin{array}{lllll}
9 & .0000 & .0000 & .0000
\end{array} \\
& \begin{array}{lllll}
10 & .0000 & .0000 & .0000
\end{array} \\
& \begin{array}{llll}
11 & -.4970 & .0000 & .0000
\end{array} \\
& \begin{array}{llll}
12 & .0000 & -1.2423 & .0000
\end{array} \\
& \begin{array}{llll}
13 & .0000 & 1.8502 & .0000
\end{array} \\
& \begin{array}{llll}
14 & .0000 & .0000 & -.6878
\end{array} \\
& \begin{array}{llll}
15 & .0000 & .1257 & .0000
\end{array} \\
& \begin{array}{llll}
16 & .0000 & .0000 & .4770
\end{array} \\
& \begin{array}{llll}
17 & .0000 & 1.3509 & .0000
\end{array} \\
& \begin{array}{llll}
18 & .5554 & .0000 & .0000
\end{array} \\
& \begin{array}{llll}
19 & .0000 & .0000 & -1.8579
\end{array} \\
& \begin{array}{llll}
20 & .0000 & .0000 & -.5452
\end{array} \\
& \begin{array}{llll}
21 & .0000 & .2436 & .0000
\end{array} \\
& \begin{array}{llll}
22 & .3268 & .0000 & .0000
\end{array} \\
& \begin{array}{llll}
23 & .0000 & .0000 & .0000
\end{array} \\
& \begin{array}{lllll}
24 & .0000 & -.0446 & .0000
\end{array} \\
& \begin{array}{lllll}
25 & -1.9254 & .0000 & .0000
\end{array} \\
& \begin{array}{llll}
26 & .0000 & .2739 & .0000
\end{array} \\
& \begin{array}{llll}
27 & .0000 & .0000 & .2143
\end{array} \\
& \begin{array}{lllll}
28 & .0000 & .0000 & .0000
\end{array} \\
& \begin{array}{llll}
29 & -.8064 & .0000 & .0000
\end{array} \\
& \begin{array}{llll}
30 & .0000 & .0000 & .0000
\end{array}
\end{aligned}
$$

$<0 \mid$ del $|\mathrm{b}\rangle *\langle\mathrm{~b}|$ rxdel $|0\rangle(\mathrm{Au})$, Rotatory

Strengths $(\mathrm{R})$ in

cgs (10**-40 erg-esu-cm/Gauss)

$$
\begin{array}{ccccc}
\text { state } & \mathrm{X} & \mathrm{Y} & \mathrm{Z} & \multicolumn{2}{c}{\mathrm{R} \text { (velocity) }} \\
1 & .0000 & .0000 & .0000 & .0000 \\
2 & .0000 & .0000 & .0000 & .0000 \\
3 & .0000 & .0000 & .0000 & .0000 \\
4 & .0000 & .0000 & .0000 & .0000 \\
5 & .0000 & .0000 & .0000 & .0000 \\
6 & .0000 & .0000 & .0000 & .0000 \\
7 & .0000 & .0000 & .0000 & .0000 \\
8 & .0000 & .0000 & .0000 & .0000 \\
9 & .0000 & .0000 & .0000 & .0000
\end{array}
$$




$\begin{array}{ccccc}10 & .0000 & .0000 & .0000 & .0000 \\ 11 & .0000 & .0000 & .0000 & .0000 \\ 12 & .0000 & .0000 & .0000 & .0000 \\ 13 & .0000 & .0000 & .0000 & .0000 \\ 14 & .0000 & .0000 & .0000 & .0000 \\ 15 & .0000 & .0000 & .0000 & .0000 \\ 16 & .0000 & .0000 & .0000 & .0000 \\ 17 & .0000 & .0000 & .0000 & .0000 \\ 18 & .0000 & .0000 & .0000 & .0000 \\ 19 & .0000 & .0000 & .0000 & .0000 \\ 20 & .0000 & .0000 & .0000 & .0000 \\ 21 & .0000 & .0000 & .0000 & .0000 \\ 22 & .0000 & .0000 & .0000 & .0000 \\ 23 & .0000 & .0000 & .0000 & .0000 \\ 24 & .0000 & .0000 & .0000 & .0000 \\ 25 & .0000 & .0000 & .0000 & .0000 \\ 26 & .0000 & .0000 & .0000 & .0000 \\ 27 & .0000 & .0000 & .0000 & .0000 \\ 28 & .0000 & .0000 & .0000 & .0000 \\ 29 & .0000 & .0000 & .0000 & .0000 \\ 30 & .0000 & .0000 & .0000 & .0000 \\ <0|\mathrm{r}| \mathrm{b}> & *<\mathrm{b}|\mathrm{rxdel}| 0> & (\mathrm{Au}), \mathrm{Rotatory}\end{array}$

Strengths $(\mathrm{R})$ in

$\begin{array}{ccccc}\text { cgs }(10 * *-40 & \text { erg-esu-cm/Gauss }) \\ \text { state } & \mathrm{X} & \mathrm{Y} & \mathrm{Z} & \mathrm{R}(\text { length }) \\ 1 & .0000 & .0000 & .0000 & .0000 \\ 2 & .0000 & .0000 & .0000 & .0000 \\ 3 & .0000 & .0000 & .0000 & .0000 \\ 4 & .0000 & .0000 & .0000 & .0000 \\ 5 & .0000 & .0000 & .0000 & .0000 \\ 6 & .0000 & .0000 & .0000 & .0000 \\ 7 & .0000 & .0000 & .0000 & .0000 \\ 8 & .0000 & .0000 & .0000 & .0000 \\ 9 & .0000 & .0000 & .0000 & .0000 \\ 10 & .0000 & .0000 & .0000 & .0000 \\ 11 & .0000 & .0000 & .0000 & .0000 \\ 12 & .0000 & .0000 & .0000 & .0000 \\ 13 & .0000 & .0000 & .0000 & .0000 \\ 14 & .0000 & .0000 & .0000 & .0000 \\ 15 & .0000 & .0000 & .0000 & .0000 \\ 16 & .0000 & .0000 & .0000 & .0000 \\ 17 & .0000 & .0000 & .0000 & .0000 \\ 18 & .0000 & .0000 & .0000 & .0000 \\ 19 & .0000 & .0000 & .0000 & .0000 \\ 20 & .0000 & .0000 & .0000 & .0000 \\ 21 & .0000 & .0000 & .0000 & .0000\end{array}$

$$
\begin{array}{ccccc}
22 & .0000 & .0000 & .0000 & .0000 \\
23 & .0000 & .0000 & .0000 & .0000 \\
24 & .0000 & .0000 & .0000 & .0000 \\
25 & .0000 & .0000 & .0000 & .0000 \\
26 & .0000 & .0000 & .0000 & .0000 \\
27 & .0000 & .0000 & .0000 & .0000 \\
28 & .0000 & .0000 & .0000 & .0000 \\
29 & .0000 & .0000 & .0000 & .0000 \\
30 & .0000 & .0000 & .0000 & .0000 \\
<0|\mathrm{del}| \mathrm{b}> & *<\mathrm{b}|\mathrm{r}| 0> & (\mathrm{Au}) & \\
\mathrm{state} & \mathrm{X} & \mathrm{Y} & \mathrm{Z} & \mathrm{O} s \mathrm{sc} .(\mathrm{frdel}) \\
1 & .0000 & .0000 & .0000 & .0000 \\
2 & .0000 & .0070 & .0000 & -.0047 \\
3 & .0000 & .0000 & -.2016 & .1344 \\
4 & -.0021 & .0000 & .0000 & .0014 \\
5 & .0000 & .0000 & .0000 & .0000 \\
6 & -.0019 & .0000 & .0000 & .0013 \\
7 & .0000 & .0000 & .0000 & .0000 \\
8 & .0000 & .0000 & .0000 & .0000 \\
9 & .0000 & .0000 & -.3251 & .2167 \\
10 & .0000 & .0000 & -.0121 & .0080 \\
11 & .0000 & -.0925 & .0000 & .0617 \\
12 & .0007 & .0000 & .0000 & -.0005 \\
13 & .0001 & .0000 & .0000 & -.0001 \\
14 & .0000 & .0000 & .0000 & .0000 \\
15 & .0003 & .0000 & .0000 & -.0002 \\
16 & .0000 & .0000 & .0000 & .0000 \\
17 & -.0019 & .0000 & .0000 & .0013 \\
18 & .0000 & -.0333 & .0000 & .0222 \\
19 & .0000 & .0000 & .0000 & .0000 \\
20 & .0000 & .0000 & .0000 & .0000 \\
21 & .0004 & .0000 & .0000 & -.0003 \\
22 & .0000 & -.0736 & .0000 & .0490 \\
23 & .0000 & .0000 & -.0003 & .0002 \\
24 & -.0027 & .0000 & .0000 & .0018 \\
25 & .0000 & -.7103 & .0000 & .4735 \\
26 & -.0014 & .0000 & .0000 & .0009 \\
27 & .0000 & .0000 & .0000 & .0000 \\
28 & .0000 & .0000 & -.1446 & .0964 \\
29 & .0000 & -.1645 & .0000 & .1097 \\
30 & .0000 & .0000 & -.4917 & .3278 \\
r & .06 x & .000
\end{array}
$$

Ground to excited state transition densities written to RWF 633

Excitation energies and oscillator strengths: 
Excited State 1: Singlet-A2 3.9521

eV $313.72 \mathrm{~nm} \mathrm{f}=0.0000$

$$
\begin{array}{ll}
20->22 & .65652 \\
20->25 & -.22255
\end{array}
$$

This state for optimization and/or second-order correction.

Copying the excited state density for this state as the 1-particle RhoCI density.

$$
\text { Excited State 2: Singlet-B2 } 4.0236
$$$$
\mathrm{eV} 308.15 \mathrm{~nm} \mathrm{f}=0.0289
$$$$
21 \text {-> } 22 \quad .69894
$$

Excited State 3: Singlet-A1 7.7051 $\mathrm{eV} 160.91 \mathrm{~nm} \mathrm{f}=0.5730$

$19->22 \quad .67812$

$21->23 \quad .10612$

Excited State 4: Singlet-B1 8.2297 $\mathrm{eV} 150.65 \mathrm{~nm} \mathrm{f}=0.0011$

18 -> $22 \quad .68090$

Excited State 5: Singlet-A2 8.6307 $\mathrm{eV} 143.65 \mathrm{~nm} \mathrm{f}=0.0000$

$11 \rightarrow 22 \quad .11088$

$14->22 \quad .52794$

$15->23 \quad .10690$

$20->22 \quad-.17984$

$20->25 \quad-.36139$

Excited State 6: Singlet-B1 8.6387 eV $143.52 \mathrm{~nm} \mathrm{f}=0.0013$

$$
\begin{array}{ll}
13->25 & -.13110 \\
15->22 & .63969 \\
15->25 & -.12819 \\
20->23 & -.15176
\end{array}
$$

Excited State 7: Singlet-A2 9.4991 $\mathrm{eV} 130.52 \mathrm{~nm} \mathrm{f}=0.0000$

$13->23 \quad-.10171$

$14->22 \quad .10345$

$15 \rightarrow 23 \quad .11573$

$16->22 \quad .61173$

$18->23 \quad-.12879$

$21->24 \quad-.21769$
Excited State 8: Singlet-A2 9.5631 $\mathrm{eV} 129.65 \mathrm{~nm} \mathrm{f}=0.0000$

$$
\begin{array}{lr}
16->22 & .21693 \\
19->26 & -.10895 \\
21->24 & .64369
\end{array}
$$

Excited State 9: Singlet-A1 9.5641

eV $129.63 \mathrm{~nm} \mathrm{f}=0.5939$

$$
\begin{array}{ll}
17->22 & -.15138 \\
21->23 & .66659
\end{array}
$$

Excited State 10: Singlet-A1

$10.0784 \mathrm{eV} 123.02 \mathrm{~nm} \mathrm{f}=0.0288$

$$
\begin{array}{cc}
17->22 & .65119 \\
19->25 & -.10836 \\
21>23 & .14102
\end{array}
$$

Excited State 11: Singlet-B2

$10.3941 \mathrm{eV} 119.28 \mathrm{~nm} \mathrm{f}=0.1591$

$$
19->23 \quad-.20339
$$$$
21->25 \quad .65290
$$

Excited State 12: Singlet-B1 $10.6185 \mathrm{eV} 116.76 \mathrm{~nm} \mathrm{f}=0.0001$

$$
\begin{array}{ll}
13->22 & -.17808 \\
14->23 & .10341 \\
15->25 & .12929 \\
19->24 & -.25130 \\
20->23 & -.24708 \\
21->26 & .26152 \\
21->28 & .42290 \\
21->30 & .19088
\end{array}
$$

Excited State 13: Singlet-B1

$10.7203 \mathrm{eV} 115.65 \mathrm{~nm} \mathrm{f}=0.0001$
$12 \rightarrow 22 \quad .11810$
$13 \rightarrow>22 \quad .29928$
$14->23 \quad-.16085$
$15->25 \quad-.20651$
$19->24 \quad-.11606$
$20->23 \quad .39720$
$21>28 \quad .32639$
21 -> $30 \quad .12538$ 
Excited State 14: Singlet-A2

$\begin{array}{cl}11.1259 \mathrm{eV} & 111.44 \mathrm{~nm} \mathrm{f}=0.0000 \\ 14->22 & -.22909 \\ 20->22 & -.10319 \\ 20->25 & -.39386 \\ 21->27 & .45547 \\ 21->29 & -.13997\end{array}$

Excited State 15: Singlet-B1

$11.2176 \mathrm{eV} 110.53 \mathrm{~nm} \mathrm{f}=0.0000$

19 -> $24 \quad-.16647$

$20->23 \quad .12488$

$21->26 \quad .56852$

$21->28 \quad-.26364$

21 -> $30 \quad-.19820$

Excited State 16: Singlet-A2

$11.3190 \mathrm{eV} 109.54 \mathrm{~nm} \mathrm{f}=0.0000$

$14->22 \quad .28306$

$20->25 \quad .37623$

$21->27 \quad .46413$

$21->29 \quad-.13030$

Excited State 17: Singlet-B1

$11.9435 \mathrm{eV} 103.81 \mathrm{~nm} \mathrm{f}=0.0017$

$12->22 \quad-.12218$

$13->22 \quad .44082$

$15->22 \quad-.13098$

$15->25 \quad-.12070$

$16->23 \quad-.26816$

$20->23 \quad-.39457$

Excited State 18: Singlet-B2

$11.9789 \mathrm{eV} 103.50 \mathrm{~nm} \mathrm{f}=0.0558$

$17->23 \quad-.13626$

$19->23 \quad .63810$

$21->25 \quad .21635$

Excited State 19: Singlet-A2 $12.1085 \mathrm{eV} 102.39 \mathrm{~nm} \mathrm{f}=0.0000$

$11->22 \quad-.21491$

$16->22 \quad .10376$

$18->23 \quad .61524$

$21->29 \quad-.11887$
Excited State 20: Singlet-A2

$12.2959 \mathrm{eV} 100.83 \mathrm{~nm} \mathrm{f}=0.0000$

18 -> $23 \quad .13596$

$19->28 \quad-.14429$

$21 \rightarrow 27 \quad .16008$

$21->29 \quad .61768$

21 -> $31 \quad .13489$

Excited State 21: Singlet-B1

$12.4250 \mathrm{eV} \quad 99.79 \mathrm{~nm} \mathrm{f}=0.0000$

19 -> $27 \quad .24874$

$21->28 \quad-.27830$

$21->30 \quad .56644$

Excited State 22: Singlet-B2

$12.6067 \mathrm{eV} \quad 98.35 \mathrm{~nm} \mathrm{f}=0.0713$

$$
\begin{array}{lr}
20->24 & .61236 \\
20->27 & .18462 \\
20->29 & -.16984 \\
20->31 & .15288
\end{array}
$$

Excited State 23: Singlet-A1

$13.0650 \mathrm{eV} \quad 94.90 \mathrm{~nm} \mathrm{f}=0.0003$

17 -> $22 \quad .15372$

19 -> $25 \quad .67069$

Excited State 24: Singlet-B1

$13.5046 \mathrm{eV} 91.81 \mathrm{~nm} \mathrm{f}=0.0007$

19 -> $24 \quad .59801$

$21 \rightarrow 26 \quad .29702$

$21->28 \quad .12415$

$21->30 \quad .13422$

Excited State 25: Singlet-B2

$13.8533 \mathrm{eV} \quad 89.50 \mathrm{~nm} \mathrm{f}=0.5223$

$20->27 \quad .58324$

$20->31 \quad-.29326$

Excited State 26: Singlet-B1

$13.8582 \mathrm{eV} \quad 89.47 \mathrm{~nm} \mathrm{f}=0.0009$

$\begin{array}{lr}12->22 & .55751 \\ 13->25 & -.10328 \\ 15->22 & -.10920 \\ 15->25 & -.11083 \\ 16->23 & .28068 \\ 20->23 & -.23135\end{array}$ 
Excited State 27: Singlet-A2

$13.9331 \mathrm{eV} 88.99 \mathrm{~nm} \mathrm{f}=0.0000$

17 -> $30 \quad-.10356$

$19->26 \quad-.26808$

$19->28 \quad .30936$

19 -> $30 \quad .35503$

$21->29 \quad .17808$

$21 \rightarrow 31 \quad-.18119$

$21->32 \quad-.31243$

Excited State 28: Singlet-A1

$14.0027 \mathrm{eV} \quad 88.54 \mathrm{~nm} \mathrm{f}=0.1174$

$20->26 \quad .18674$

$20->28 \quad .64430$
Excited State 29: Singlet-B2

$14.3540 \mathrm{eV} \quad 86.38 \mathrm{~nm} \mathrm{f}=0.1554$

$20->24 \quad .23976$

$20->29 \quad .59100$

20 -> $31 \quad-.19204$

Excited State 30: Singlet-A1

$14.5716 \mathrm{eV} 85.09 \mathrm{~nm} \mathrm{f}=0.3280$

$16->28 \quad-.14289$

$18 \rightarrow 24 \quad .61888$

$20->26 \quad-.13903$

20 -> $30 \quad .10682$ 
Prediction of Excited States for $\mathbf{1 a}\left(\mathbf{C}_{\mathbf{2 v}}\right)$ :

The following Gaussian input file gave the output below for the single excitations within the CIS method. The geometry used was HF optimized as described above.

\section{Output File:}

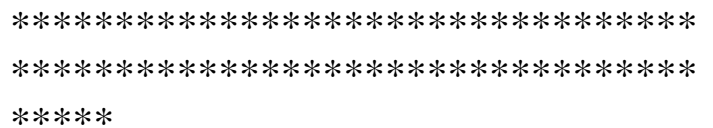

Excited states from <AA,BB:AA,BB> singles matrix:

\begin{tabular}{|c|c|c|}
\hline \multicolumn{3}{|c|}{$\begin{array}{l}\text { Ground to excited state Transition } \\
\text { electric dipole moments }(\mathrm{Au}) \text { : }\end{array}$} \\
\hline state & $X$ & Z Osc. \\
\hline 1 & $.0000-.8464$ & $.0000 \quad .0570$ \\
\hline 2 & .0000 .0000 & $.0000 \quad .0000$ \\
\hline 3 & .0152 .0000 & $.0000 \quad .0000$ \\
\hline 4 & .0000 .0000 & $\begin{array}{ll}.0000 & .0000\end{array}$ \\
\hline 5 & -.1083 .0000 & $.0000 \quad .0017$ \\
\hline 6 & .0000 .0000 & $\begin{array}{ll}.0000 & .0000\end{array}$ \\
\hline 7 & .0156 .0000 & $\begin{array}{ll}.0000 & .0000\end{array}$ \\
\hline 8 & .0000 $\quad .0000$ & $\begin{array}{ll}.0000 & .0000\end{array}$ \\
\hline 9 & $\begin{array}{ll}-.0946 & .0000\end{array}$ & $.0000 \quad .0014$ \\
\hline 10 & .0000 .1375 & $.0000 \quad .0029$ \\
\hline 11 & $.0000 \quad .0000$ & $.8118 \quad .1005$ \\
\hline 12 & $.0000 \quad .0000$ & $.0000 \quad .0000$ \\
\hline 13 & $.0000 \quad .6892$ & $.0000 \quad .0731$ \\
\hline 14 & .0000 .0000 & $.1820 \quad .0052$ \\
\hline 15 & $.0000 \quad .5054$ & $.0000 \quad .0412$ \\
\hline 16 & .0000 .0000 & $-.2825 \quad .0129$ \\
\hline 17 & .0000 .2536 & $.0000 \quad .0108$ \\
\hline 18 & .0000 .0000 & $-1.4646 \quad .3653$ \\
\hline 19 & .0000 .0000 & 2.51971 .0990 \\
\hline 20 & $-.1072 \quad .0000$ & $.0000 \quad .0021$ \\
\hline 21 & .0000 .0000 & $.0000 \quad .0000$ \\
\hline 22 & .0000 .0000 & $.0000 \quad .0000$ \\
\hline 23 & .0000 .0000 & 2.66081 .3720 \\
\hline 24 & $.0000-.6128$ & $.0000 \quad .0728$ \\
\hline 25 & .0000 .0000 & $.0000 \quad .0000$ \\
\hline
\end{tabular}

$\begin{array}{lllll}26 & .1538 & .0000 & .0000 & .0047 \\ 27 & .0000 & -3.3271 & .0000 & 2.1926 \\ 28 & -.3967 & .0000 & .0000 & .0312 \\ 29 & .0000 & 2.9683 & .0000 & 1.7838 \\ 30 & .0000 & .0000 & .0000 & .0000\end{array}$

Ground to excited state transition velocity dipole Moments (Au):

\begin{tabular}{ccccc} 
state & $\mathrm{X}$ & $\mathrm{Y}$ & $\mathrm{Z}$ & \multicolumn{2}{c}{ Osc. } \\
1 & .0000 & .0619 & .0000 & .0214 \\
2 & .0000 & .0000 & .0000 & .0000 \\
3 & .0027 & .0000 & .0000 & .0000 \\
4 & .0000 & .0000 & .0000 & .0000 \\
5 & .0027 & .0000 & .0000 & .0000 \\
6 & .0000 & .0000 & .0000 & .0000 \\
7 & -.0035 & .0000 & .0000 & .0000 \\
8 & .0000 & .0000 & .0000 & .0000 \\
9 & .0173 & .0000 & .0000 & .0009 \\
10 & .0000 & -.0279 & .0000 & .0023 \\
11 & .0000 & .0000 & -.1451 & .0614 \\
12 & .0000 & .0000 & .0000 & .0000 \\
13 & .0000 & -.1475 & .0000 & .0629 \\
14 & .0000 & .0000 & -.0286 & .0023 \\
15 & .0000 & -.0845 & .0000 & .0196 \\
16 & .0000 & .0000 & .0229 & .0014 \\
17 & .0000 & -.0402 & .0000 & .0043 \\
18 & .0000 & .0000 & .1817 & .0862 \\
19 & .0000 & .0000 & -.3059 & .2403 \\
20 & .0318 & .0000 & .0000 & .0025 \\
21 & .0000 & .0000 & .0000 & .0000 \\
22 & .0000 & .0000 & .0000 & .0000 \\
23 & .0000 & .0000 & -.4925 & .5563 \\
24 & .0000 & .1126 & .0000 & .0291 \\
25 & .0000 & .0000 & .0000 & .0000 \\
26 & -.0232 & .0000 & .0000 & .0012 \\
27 & .0000 & .5560 & .0000 & .6936 \\
28 & .0355 & .0000 & .0000 & .0028 \\
29 & .0000 & -.4693 & .0000 & .4835 \\
30 & .0000 & .0000 & .0000 & .0000
\end{tabular}


Ground to excited state transition

magnetic dipole Moments $(\mathrm{Au})$ :

$$
\begin{aligned}
& \begin{array}{cccc}
\text { state } & \mathrm{X} & \mathrm{Y} & \mathrm{Z} \\
1 & .8726 & .0000 & .0000 \\
2 & .0000 & .0000 & .6398
\end{array} \\
& \begin{array}{lllll}
2 & .0000 & .0000 & .6398
\end{array} \\
& \begin{array}{lllll}
3 & .0000 & -.3067 & .0000
\end{array} \\
& \begin{array}{lllll}
4 & .0000 & .0000 & -.3487
\end{array} \\
& \begin{array}{lllll}
5 & .0000 & .0181 & .0000
\end{array} \\
& \begin{array}{lllll}
6 & .0000 & .0000 & -.0551
\end{array} \\
& \begin{array}{lllll}
7 & .0000 & .1452 & .0000
\end{array} \\
& \begin{array}{lllll}
8 & .0000 & .0000 & .0036
\end{array} \\
& \begin{array}{lllll}
9 & .0000 & .0959 & .0000
\end{array} \\
& \begin{array}{lllll}
10 & .0544 & .0000 & .0000
\end{array} \\
& \begin{array}{llll}
11 & .0000 & .0000 & .0000
\end{array} \\
& \begin{array}{lllll}
12 & .0000 & .0000 & -.0412
\end{array} \\
& \begin{array}{lllll}
13 & .2357 & .0000 & .0000
\end{array} \\
& \begin{array}{lllll}
14 & .0000 & .0000 & .0000
\end{array} \\
& \begin{array}{llll}
15 & .007497 & .0000 & .0000
\end{array} \\
& \begin{array}{lllll}
16 & .0000 & .0000 & .0000
\end{array} \\
& \begin{array}{lllll}
17 & -.0120 & .0000 & .0000
\end{array} \\
& \begin{array}{lllll}
18 & .0000 & .0000 & .0000
\end{array} \\
& \begin{array}{lllll}
19 & .0000 & .0000 & .0000
\end{array} \\
& \begin{array}{lllll}
20 & .0000 & -.1789 & .0000
\end{array} \\
& \begin{array}{lllll}
21 & .0000 & .0000 & .2192
\end{array} \\
& \begin{array}{lllll}
22 & .0000 & .0000 & -.4530
\end{array} \\
& \begin{array}{lllll}
23 & .0000 & .0000 & .0000
\end{array} \\
& \begin{array}{lllll}
24 & -.0900 & .0000 & .0000
\end{array} \\
& \begin{array}{lllll}
25 & .0000 & .0000 & -.2561
\end{array} \\
& \begin{array}{lllll}
26 & .0000 & -.7822 & .0000
\end{array} \\
& \begin{array}{lllll}
27 & -.7395 & .0000 & .0000
\end{array} \\
& \begin{array}{llll}
28 & .0000 & 2.0197 & .0000
\end{array} \\
& \begin{array}{llll}
29 & .6393 & .0000 & .0000
\end{array} \\
& \begin{array}{lllll}
30 & 0000 & 0000 & 1.8958
\end{array}
\end{aligned}
$$$$
<0|\operatorname{del}| \mathrm{b}\rangle *<\mathrm{b}|\mathrm{rxdel}| 0\rangle(\mathrm{Au}), \text { Rotatory }
$$$$
\text { Strengths }(\mathrm{R}) \text { in }
$$$$
\text { cgs (10**-40 erg-esu-cm/Gauss) }
$$$$
\begin{array}{ccccc}
\text { state } & \mathrm{X} & \mathrm{Y} & \mathrm{Z} & \multicolumn{2}{c}{\mathrm{R} \text { (velocity) }} \\
1 & .0000 & .0000 & .0000 & .0000 \\
2 & .0000 & .0000 & .0000 & .0000 \\
3 & .0000 & .0000 & .0000 & .0000 \\
4 & .0000 & .0000 & .0000 & .0000 \\
5 & .0000 & .0000 & .0000 & .0000 \\
6 & .0000 & .0000 & .0000 & .0000 \\
7 & .0000 & .0000 & .0000 & .0000 \\
8 & .0000 & .0000 & .0000 & .0000 \\
9 & .0000 & .0000 & .0000 & .0000
\end{array}
$$

$$
\begin{array}{ccccc}
10 & .0000 & .0000 & .0000 & .0000 \\
11 & .0000 & .0000 & .0000 & .0000 \\
12 & .0000 & .0000 & .0000 & .0000 \\
13 & .0000 & .0000 & .0000 & .0000 \\
14 & .0000 & .0000 & .0000 & .0000 \\
15 & .0000 & .0000 & .0000 & .0000 \\
16 & .0000 & .0000 & .0000 & .0000 \\
17 & .0000 & .0000 & .0000 & .0000 \\
18 & .0000 & .0000 & .0000 & .0000 \\
19 & .0000 & .0000 & .0000 & .0000 \\
20 & .0000 & .0000 & .0000 & .0000 \\
21 & .0000 & .0000 & .0000 & .0000 \\
22 & .0000 & .0000 & .0000 & .0000 \\
23 & .0000 & .0000 & .0000 & .0000 \\
24 & .0000 & .0000 & .0000 & .0000 \\
25 & .0000 & .0000 & .0000 & .0000 \\
26 & .0000 & .0000 & .0000 & .0000 \\
27 & .0000 & .0000 & .0000 & .0000 \\
28 & .0000 & .0000 & .0000 & .0000 \\
29 & .0000 & .0000 & .0000 & .0000 \\
30 & .0000 & .0000 & .0000 & .0000 \\
<0|\mathrm{r}| \mathrm{b}> & *<\mathrm{b}|\mathrm{rxdel}| 0> & (\mathrm{Au}), \text { Rotatory } \\
\mathrm{Str} & &
\end{array}
$$$$
\text { Strengths }(\mathrm{R}) \text { in }
$$$$
\text { cgs }(10 * *-40 \text { erg-esu-cm/Gauss) }
$$$$
\begin{array}{lllll}
\text { state } & \mathrm{X} & \mathrm{Y} & \mathrm{Z} & \mathrm{R} \text { (length) }
\end{array}
$$$$
\begin{array}{llllll}
1 & .0000 & .0000 & .0000 & .0000
\end{array}
$$$$
\begin{array}{llllll}
2 & .0000 & .0000 & .0000 & .0000
\end{array}
$$$$
\begin{array}{llllll}
3 & .0000 & .0000 & .0000 & .0000
\end{array}
$$$$
\begin{array}{llllll}
4 & .0000 & .0000 & .0000 & .0000
\end{array}
$$$$
\begin{array}{llllll}
5 & .0000 & .0000 & .0000 & .0000
\end{array}
$$$$
\begin{array}{llllll}
6 & .0000 & .0000 & .0000 & .0000
\end{array}
$$$$
\begin{array}{llllll}
7 & .0000 & .0000 & .0000 & .0000
\end{array}
$$$$
\begin{array}{llllll}
8 & .0000 & .0000 & .0000 & .0000
\end{array}
$$$$
\begin{array}{llllll}
9 & .0000 & .0000 & .0000 & .0000
\end{array}
$$$$
\begin{array}{llllll}
10 & .0000 & .0000 & .0000 & .0000
\end{array}
$$$$
\begin{array}{llllll}
11 & .0000 & .0000 & .0000 & .0000
\end{array}
$$$$
\begin{array}{llllll}
12 & .0000 & .0000 & .0000 & .0000
\end{array}
$$$$
\begin{array}{llllll}
13 & .0000 & .0000 & .0000 & .0000
\end{array}
$$$$
\begin{array}{llllll}
14 & .0000 & .0000 & .0000 & .0000
\end{array}
$$$$
\begin{array}{llllll}
15 & .0000 & .0000 & .0000 & .0000
\end{array}
$$$$
\begin{array}{lllll}
16 & .0000 & .0000 & .0000 & .0000
\end{array}
$$$$
\begin{array}{llllll}
17 & .0000 & .0000 & .0000 & .0000
\end{array}
$$$$
\begin{array}{llllll}
18 & .0000 & .0000 & .0000 & .0000
\end{array}
$$$$
\begin{array}{llllll}
19 & .0000 & .0000 & .0000 & .0000
\end{array}
$$$$
\begin{array}{llllll}
20 & .0000 & .0000 & .0000 & .0000
\end{array}
$$$$
\begin{array}{llllll}
21 & .0000 & .0000 & .0000 & .0000
\end{array}
$$ 


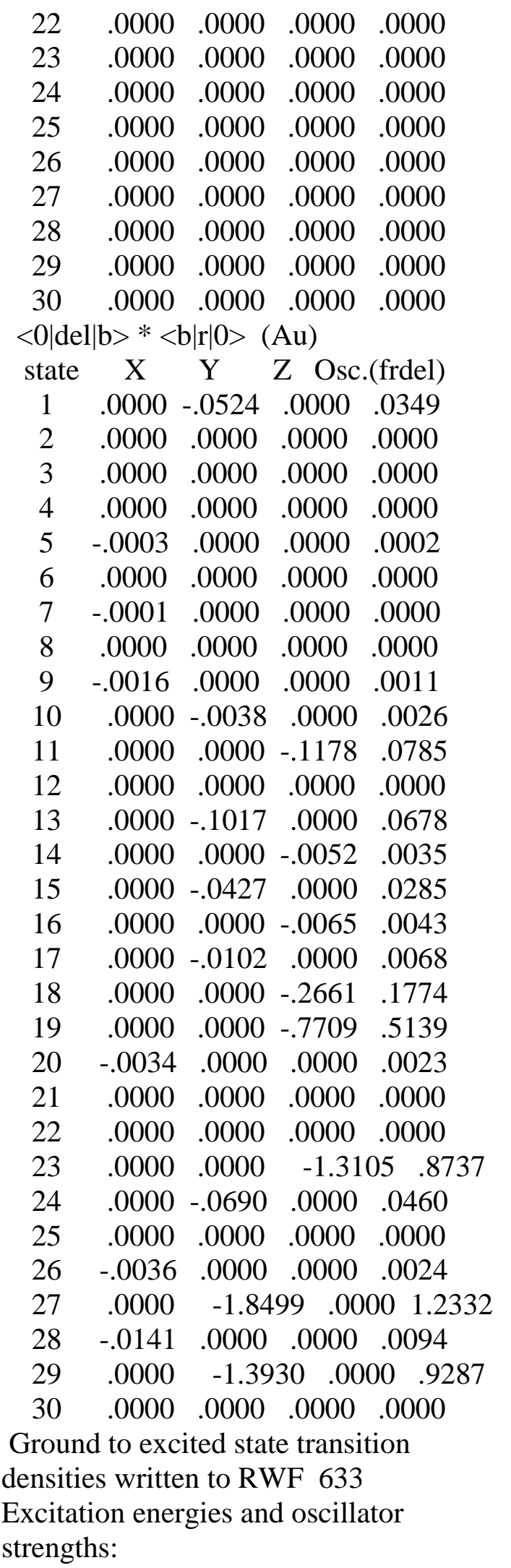
Excited State 1: Singlet-B2 3.2500 $\mathrm{eV} 381.49 \mathrm{~nm} \mathrm{f}=0.0570$ $101->102 \quad .68999$

This state for optimization and/or second-order correction.

Copying the excited state density for this state as the 1-particle RhoCI density.
Excited State 2: Singlet-A2 3.6594 $\mathrm{eV} 338.81 \mathrm{~nm} \mathrm{f}=0.0000$

$$
\begin{array}{cc}
92->102 & .55235 \\
92->117 & .16905 \\
95->102 & .26362 \\
100->102 & .22420
\end{array}
$$

Excited State 3: Singlet-B1 5.5613 $\mathrm{eV} 222.94 \mathrm{~nm} \mathrm{f}=0.0000$

$$
\begin{array}{ll}
90->102 & .17400 \\
96->102 & .56146 \\
99->102 & .31149
\end{array}
$$
Excited State 4: Singlet-A2 5.6603 eV $219.04 \mathrm{~nm} \mathrm{f}=0.0000$

$$
\begin{array}{cc}
89->102 & .13939 \\
92->102 & -.14093 \\
95->102 & -.11865 \\
100->102 & .63551
\end{array}
$$

Excited State 5: Singlet-B1 5.8807 eV $210.83 \mathrm{~nm} \mathrm{f}=0.0017$

$$
\begin{array}{lr}
86->102 & .11681 \\
96->102 & -.29980 \\
99->102 & .57635
\end{array}
$$

Excited State 6: Singlet-A2 6.0424 eV $205.19 \mathrm{~nm} \mathrm{f}=0.0000$

$$
\begin{array}{ll}
81->102 & -.10914 \\
92->102 & -.14718 \\
93->103 & -.18767 \\
93->108 & -.11017 \\
94->105 & -.19632 \\
95->102 & .39775 \\
95->104 & .22011 \\
95->109 & -.11122 \\
96->107 & .16294 \\
96->110 & -.17047
\end{array}
$$




$$
\begin{aligned}
& 97->103 \quad-.11443 \\
& 98->105 \quad-.12115 \\
& 99->107 \quad-.11927 \\
& 100 \text {->104 -.14034 } \\
& \text { Excited State 7: Singlet-B1 } \\
& \text { eV } 201.76 \mathrm{~nm} \mathrm{f}=0.0000 \\
& 93->105 \quad-.13837 \\
& 93->106 \quad-.11628 \\
& 94->108 \quad-.15846 \\
& 95->110 \quad-.15032 \\
& 96->104 \quad .13283 \\
& 96->109 \quad-.12302 \\
& 97->105 \quad-.12544 \\
& 97->106 \quad .27198 \\
& 98->103 \quad-.20504 \\
& 98->108 \quad .20323 \\
& 99->104 \quad-.19180 \\
& 99->109 \quad-.22812 \\
& 100->107 \quad-.26003 \\
& 100->110 \quad-.16330
\end{aligned}
$$

Excited State 8: Singlet-A2 eV $201.23 \mathrm{~nm} \mathrm{f}=0.0000$

$$
\begin{array}{cc}
93->103 & .10088 \\
94->106 & -.11233 \\
95->102 & -.10634 \\
95->104 & -.10627 \\
97->103 & -.13649 \\
97->108 & .28280 \\
98->106 & .31393 \\
99->107 & -.21827 \\
99->110 & -.23015 \\
100->104 & -.15047 \\
100->109 & -.29246
\end{array}
$$

Excited State 9: Singlet-B1 6.2045 eV $199.83 \mathrm{~nm} \mathrm{f}=0.0014$

$$
\begin{array}{ll}
93->105 & -.27304 \\
94->103 & -.26832 \\
95->107 & .23779 \\
95->110 & -.12539 \\
96->102 & -.12484 \\
96->104 & .28156 \\
97->106 & -.18406 \\
98->108 & -.18646
\end{array}
$$

6.1612

$$
\begin{array}{cc}
99->102 & .16514 \\
99->109 & .17264 \\
100->110 & .17098
\end{array}
$$

Excited State 10: Singlet-B2 6.2105 eV $199.64 \mathrm{~nm} \mathrm{f}=0.0029$

$$
\begin{array}{cc}
93->107 & .11936 \\
93->110 & -.16326 \\
94->102 & .11597 \\
94->104 & .15565 \\
94->109 & -.14069 \\
95->105 & .24964 \\
95->106 & .11983 \\
96->103 & .23648 \\
96->108 & .15280 \\
97->107 & .18666 \\
98->104 & .18661 \\
98->109 & .10663 \\
99->103 & -.18208 \\
99->108 & .16710 \\
100->105 & -.16002 \\
100->106 & .22058
\end{array}
$$

Excited State 11: Singlet-A1 6.2238 $\mathrm{eV} 199.21 \mathrm{~nm} \mathrm{f}=0.1005$

$$
\begin{array}{cc}
93->104 & .13566 \\
93->109 & -.12060 \\
94->110 & -.15741 \\
95->103 & .18723 \\
95->108 & .15728 \\
96->105 & .22904 \\
96->106 & .10787 \\
97->104 & .16990 \\
97->109 & .14811 \\
98->107 & .20709 \\
99->105 & -.12913 \\
99->106 & .25576 \\
100->103 & -.22492 \\
100->108 & .19818
\end{array}
$$

Excited State 12: Singlet-A2 6.2809 $\mathrm{eV} 197.40 \mathrm{~nm} \mathrm{f}=0.0000$
$81->102-.12236$
$92->102 \quad-.14257$
$93->103 \quad .21211$
$94->105 \quad .21664$ 


$$
\begin{array}{lr}
95->102 & .44254 \\
95->104 & -.20521 \\
96->107 & -.18082 \\
96->110 & .11775 \\
98->105 & .10665
\end{array}
$$

Excited State 13: Singlet-B2 6.2820 eV $197.36 \mathrm{~nm} \mathrm{f}=0.0731$

$$
\begin{array}{cc}
93->107 & -.17691 \\
94->104 & -.20694 \\
95->105 & -.22817 \\
96->103 & -.23753 \\
97->107 & .11108 \\
97->110 & .19118 \\
98->102 & -.18752 \\
98->109 & .20867 \\
99->108 & .24954 \\
100->106 & .29770
\end{array}
$$

Excited State 14: Singlet-A1 6.3615 eV $194.90 \mathrm{~nm} \mathrm{f}=0.0052$

$$
\begin{array}{ll}
93->104 & .23647 \\
94->107 & .21161 \\
95->103 & .24699 \\
96->105 & .24918 \\
97->102 & .23171 \\
97->109 & -.19782 \\
98->110 & -.19896 \\
99->106 & -.22357 \\
100->108 & -.21999
\end{array}
$$

Excited State 15: Singlet-B2

$\mathrm{eV} 188.17 \mathrm{~nm} \mathrm{f}=0.0412$

98 ->102 .67175

Excited State 16: Singlet-A1 6.5954 eV $187.99 \mathrm{~nm} \mathrm{f}=0.0129$

$97->102 \quad .65460$

99 ->106 .10462

Excited State 17: Singlet-B2 6.8806 $\mathrm{eV} 180.19 \mathrm{~nm} \mathrm{f}=0.0108$ 94 ->102 .67557

Excited State 18: Singlet-A1 6.9506 $\mathrm{eV} 178.38 \mathrm{~nm} \mathrm{f}=0.3653$

$$
\begin{array}{cc}
91->102 & -.23068 \\
93->102 & .64178
\end{array}
$$

Excited State 19: Singlet-A1 7.0656

eV $175.48 \mathrm{~nm} \mathrm{f}=1.0990$

$$
\begin{array}{ll}
91->102 & .59908 \\
93->102 & .23374 \\
93->104 & -.11804
\end{array}
$$

Excited State 20: Singlet-B1 7.3838

$\mathrm{eV} 167.91 \mathrm{~nm} \mathrm{f}=0.0021$

$$
\begin{array}{cc}
91->105 & -.13228 \\
101->103 & .65102 \\
101->130 & -.11202
\end{array}
$$

Excited State 21: Singlet-A2 7.4529

eV $166.36 \mathrm{~nm} \mathrm{f}=0.0000$

$$
\begin{array}{cc}
91->108 & -.12378 \\
101->105 & -.12551 \\
101->106 & .64305 \\
101->133 & -.11299
\end{array}
$$

Excited State 22: Singlet-A2 7.6987

$\mathrm{eV} 161.05 \mathrm{~nm} \mathrm{f}=0.0000$

$$
\begin{array}{cr}
91->103 & -.15487 \\
101->105 & .64290 \\
101->106 & .12664
\end{array}
$$

Excited State 23: Singlet-A1 7.9103

eV $156.74 \mathrm{~nm} \mathrm{f}=1.3720$

$$
91->102 \quad-.15800
$$

$93->104 \quad-.15289$

$93->109 \quad .12823$

$94->110 \quad .16902$

$95->103 \quad .11856$

$96->105 \quad .13296$

$97->104 \quad-.21107$

$97->109 \quad-.16929$

$98->107 \quad-.24887$

99 ->106 .16123

$100->103 \quad-.16714$

$100->108 \quad .12205$

$101->107 \quad .16854$

$101->112 \quad .27930$ 
Excited State 24: Singlet-B2 7.9109 eV $156.73 \mathrm{~nm} \mathrm{f}=0.0728$

$$
\begin{array}{cc}
93->107 & .15176 \\
93->110 & -.18531 \\
94->104 & .20237 \\
94->109 & -.16893 \\
95->105 & -.17879 \\
96->103 & -.18557 \\
97->107 & .25706 \\
98->104 & .26594 \\
98->109 & .13715 \\
99->103 & .14986 \\
99->108 & -.10936 \\
100->105 & .13626 \\
100->106 & -.17604
\end{array}
$$

Excited State 25: Singlet-A2 7.9573

$$
\text { eV } 155.81 \mathrm{~nm} \mathrm{f}=0.0000
$$

$$
\begin{array}{cc}
85->102 & -.12955 \\
93->103 & .19104 \\
93->108 & .12983 \\
94->105 & .20982 \\
94->106 & .12019 \\
95->104 & .20255 \\
95->109 & -.11550 \\
96->107 & .14520 \\
96->110 & -.13620 \\
97->103 & .19732 \\
97->108 & -.12817 \\
98->105 & .17215 \\
98->106 & -.17088 \\
99->107 & -.20253 \\
100->104 & -.23068 \\
100->109 & -.11964
\end{array}
$$

Excited State 26: Singlet-B1 8.0496 eV $154.03 \mathrm{~nm} \mathrm{f}=0.0047$

$$
\begin{array}{rr}
91->106 & -.14814 \\
96->104 & -.10525 \\
97->106 & .12274 \\
98->103 & -.11674 \\
99->104 & .11730 \\
100->107 & .15322 \\
101->108 & .53759
\end{array}
$$

Excited State 27: Singlet-B2 8.0847 eV $153.36 \mathrm{~nm} \mathrm{f}=2.1926$

$$
\begin{array}{cc}
93->107 & -.14475 \\
94->104 & -.12552 \\
95->105 & .12926 \\
96->103 & .14517 \\
97->107 & .10963 \\
97->110 & .14847 \\
98->109 & .17466 \\
99->108 & -.11852 \\
100->106 & -.16857 \\
101->104 & .51650 \\
101->117 & -.10354
\end{array}
$$

Excited State 28: Singlet-B1 8.1010 eV $153.05 \mathrm{~nm} \mathrm{f}=0.0312$

$$
\begin{array}{cc}
91->106 & -.10249 \\
93->105 & .14268 \\
94->103 & .11824 \\
94->108 & .16116 \\
95->110 & -.10610 \\
96->104 & .13605 \\
97->105 & .12785 \\
97->106 & -.19894 \\
98->103 & .19819 \\
98->108 & -.13328 \\
99->104 & -.17703 \\
99->109 & -.13728 \\
100->107 & -.22748 \\
101->108 & .32796
\end{array}
$$

Excited State 29: Singlet-B2 8.2640 eV $150.03 \mathrm{~nm} \mathrm{f}=1.7838$

$$
\begin{array}{cc}
93->107 & .18137 \\
94->104 & .27415 \\
95->105 & -.12790 \\
96->103 & -.16378 \\
97->110 & -.16867 \\
98->109 & -.19248 \\
99->108 & .10856 \\
100->106 & .17867 \\
101->104 & .42043
\end{array}
$$

Excited State 30: Singlet-A2 8.2989 $\mathrm{eV} 149.40 \mathrm{~nm} \mathrm{f}=0.0000$ 85 ->102 .10060 


$\begin{array}{ll}93->103 & .21534 \\ 94->105 & .21148 \\ 95->104 & .25112 \\ 96->107 & .19297 \\ 97->108 & .21390\end{array}$

$$
\begin{array}{cc}
98->106 & .24422 \\
99->107 & .13720 \\
99->110 & .16607 \\
100->109 & .21351
\end{array}
$$


Prediction of Excited States for $\mathbf{1 a}\left(\mathbf{C}_{2}\right)$ :

The following Gaussian input file gave the output below for the single excitations within the CIS method. The geometry used was HF optimized as described above.

\section{Output File:}

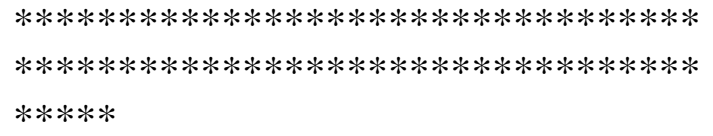

Excited states from <AA,BB:AA,BB> singles matrix:

$* * * * * * * * * * * * * * * * * * * * * * * * * * * * * * * * *$
$* * * * * * * * * * * * * * * * * * * * * * * * * * * * * * * * * * *$
$* * * * *$

Ground to excited state Transition

electric dipole moments $(\mathrm{Au})$ :

\begin{tabular}{ccccc} 
state & $\mathrm{X}$ & $\mathrm{Y}$ & $\mathrm{Z}$ & \multicolumn{2}{c}{ Osc. } \\
1 & .0484 & -.9364 & .0000 & .0690 \\
2 & .0000 & .0000 & .0631 & .0004 \\
3 & .0000 & .0000 & 2.3693 & .6973 \\
4 & -.1978 & -.9644 & .0000 & .1365 \\
5 & .0000 & .0000 & -.1822 & .0047 \\
6 & -.1781 & 1.0323 & .0000 & .1575 \\
7 & .0000 & .0000 & -.1069 & .0016 \\
8 & .0001 & -.1025 & .0000 & .0015 \\
9 & -.0126 & .6382 & .0000 & .0602 \\
10 & .0000 & .0000 & .3705 & .0205 \\
11 & .0000 & .0000 & .4451 & .0300 \\
12 & .1017 & -.1084 & .0000 & .0035 \\
13 & .0000 & .0000 & -.1966 & .0062 \\
14 & -.7112 & -.3113 & .0000 & .0992 \\
15 & .0000 & .0000 & -.9683 & .1545 \\
16 & .0812 & -.4170 & .0000 & .0304 \\
17 & .0000 & .0000 & 2.2767 & .8882 \\
18 & .0000 & .0000 & .4488 & .0348 \\
19 & -.6836 & -.5465 & .0000 & .1355 \\
20 & -.0539 & .9289 & .0000 & .1575 \\
21 & .0435 & 3.0620 & .0000 & 1.7490 \\
22 & .0000 & .0000 & -.3610 & .0245 \\
23 & .0000 & .0000 & 1.8313 & .6455 \\
24 & -1.6932 & .0224 & .0000 & .5536 \\
25 & .0000 & .0000 & -.1167 & .0026 \\
26 & .7042 & .9393 & .0000 & .2688 \\
27 & .0000 & .0000 & -1.6448 & .5411
\end{tabular}

$$
\begin{array}{rrrrr}
28 & -.3091 & 1.0923 & .0000 & .2580 \\
29 & .1802 & 1.2284 & .0000 & .3111 \\
30 & .0000 & .0000 & 1.3358 & .3694
\end{array}
$$

Ground to excited state transition velocity dipole Moments (Au):

$$
\begin{array}{ccccc}
\text { state } & \mathrm{X} & \mathrm{Y} & \mathrm{Z} & \multicolumn{2}{c}{\text { Osc. }} \\
1 & -.0015 & .0679 & .0000 & .0262 \\
2 & .0000 & .0000 & .0068 & .0002 \\
3 & .0000 & .0000 & -.2050 & .1504 \\
4 & -.0080 & .1123 & .0000 & .0400 \\
5 & .0000 & .0000 & -.0154 & .0007 \\
6 & -.0037 & -.1450 & .0000 & .0651 \\
7 & .0000 & .0000 & .0030 & .0000 \\
8 & -.0050 & -.0082 & .0000 & .0003 \\
9 & -.0059 & -.0881 & .0000 & .0235 \\
10 & .0000 & .0000 & -.0129 & .0005 \\
11 & .0000 & .0000 & -.0999 & .0293 \\
12 & .0026 & .0531 & .0000 & .0079 \\
13 & .0000 & .0000 & .0041 & .0000 \\
14 & -.0134 & .0521 & .0000 & .0078 \\
15 & .0000 & .0000 & .0543 & .0079 \\
16 & -.0072 & .0407 & .0000 & .0045 \\
17 & .0000 & .0000 & -.2939 & .2240 \\
18 & .0000 & .0000 & -.0645 & .0107 \\
19 & .0016 & .0143 & .0000 & .0005 \\
20 & -.0007 & -.1592 & .0000 & .0619 \\
21 & .0096 & -.4542 & .0000 & .4919 \\
22 & .0000 & .0000 & .0428 & .0043 \\
23 & .0000 & .0000 & -.1761 & .0716 \\
24 & .0532 & -.0451 & .0000 & .0112 \\
25 & .0000 & .0000 & -.0762 & .0133 \\
26 & -.0363 & -.1656 & .0000 & .0655 \\
27 & .0000 & .0000 & .1755 & .0684 \\
28 & .0279 & -.0435 & .0000 & .0059 \\
29 & -.0259 & -.1598 & .0000 & .0577 \\
30 & .0000 & .0000 & -.2061 & .0912
\end{array}
$$

Ground to excited state transition

magnetic dipole Moments $(\mathrm{Au})$ :

$\begin{array}{cccc}\text { state } & \mathrm{X} & \mathrm{Y} & \mathrm{Z} \\ 1 & .5755 & .0111 & .0000\end{array}$




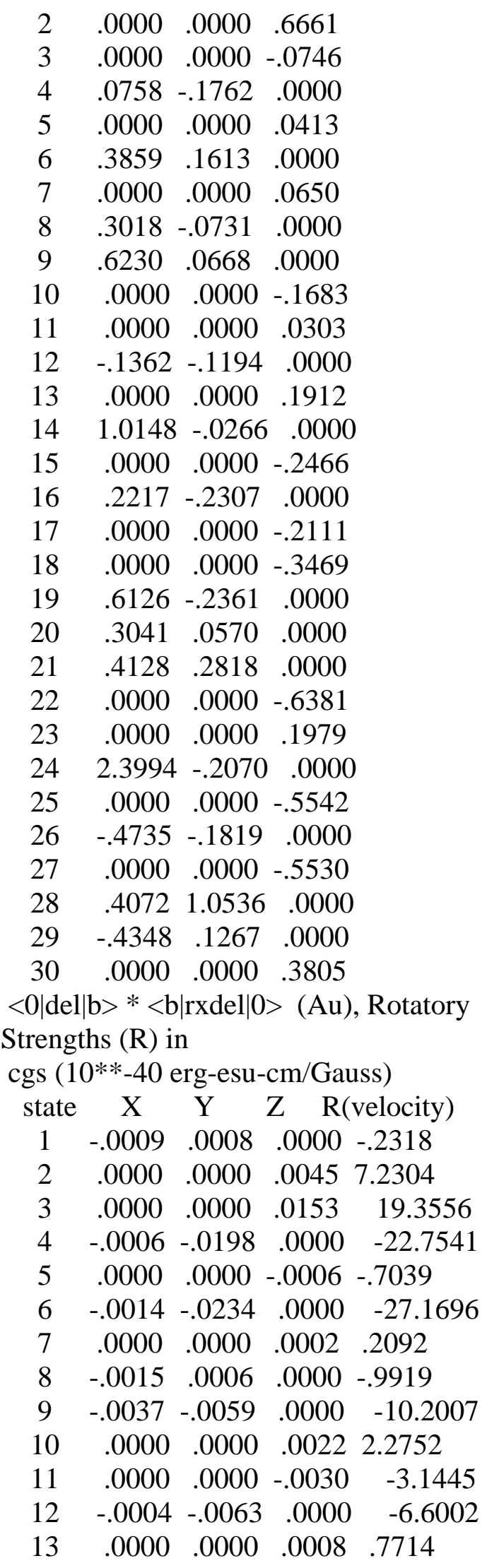

$\begin{array}{ccccc}14 & -.0136 & -.0014 & .0000 & -14.2669 \\ 15 & .0000 & .0000 & -.0134 & -12.7589 \\ 16 & -.0016 & -.0094 & .0000 & -10.2481 \\ 17 & .0000 & .0000 & .0621 & 56.9058 \\ 18 & .0000 & .0000 & .0224 & 20.3420 \\ 19 & .0010 & -.0034 & .0000 & -2.1314 \\ 20 & -.0002 & -.0091 & .0000 & -8.0170 \\ 21 & .0040 & -.1280 & .0000 & - \\ 104.5070 & & & \\ 22 & .0000 & .0000 & -.0273 & -22.7862 \\ 23 & .0000 & .0000 & -.0349 & -28.4555 \\ 24 & .1275 & .0093 & .0000 & 111.4230 \\ 25 & .0000 & .0000 & .0422 & 34.1180 \\ 26 & .0172 & .0301 & .0000 & 38.1227 \\ 27 & .0000 & .0000 & -.0970 & -76.2378 \\ 28 & .0114 & -.0458 & .0000 & -27.0499 \\ 29 & .0113 & -.0203 & .0000 & -6.9914 \\ 30 & .0000 & .0000 & -.0784 & -59.5268 \\ <0|\mathrm{r}| \mathrm{b}> & *<\mathrm{b}|\mathrm{rxdel}| 0> & (\text { Au), Rotatory }\end{array}$

$<0|\mathrm{r}| \mathrm{b}>*<\mathrm{b}|\mathrm{rxdel}| 0>(\mathrm{Au})$, Rotatory

Strengths $(\mathrm{R})$ in cgs $(10 * *$-40 erg-esu-cm/Gauss)

\begin{tabular}{ccccc} 
state & $\mathrm{X}$ & $\mathrm{Y}$ & $\mathrm{Z}$ & \multicolumn{2}{c}{$\mathrm{R}$ (length) } \\
1 & .0279 & -.0104 & .0000 & -4.1178 \\
2 & .0000 & .0000 & .0420 & -9.9048 \\
3 & .0000 & .0000 & -.1768 & 41.6747 \\
4 & -.0150 & .1699 & .0000 & -36.5099 \\
5 & .0000 & .0000 & -.0075 & 1.7761 \\
6 & -.0687 & .1665 & .0000 & -23.0354 \\
7 & .0000 & .0000 & -.0069 & 1.6367 \\
8 & .0000 & .0075 & .0000 & -1.7698 \\
9 & -.0079 & .0426 & .0000 & -8.1936 \\
10 & .0000 & .0000 & -.0624 & 14.7028 \\
11 & .0000 & .0000 & .0135 & -3.1803 \\
12 & -.0139 & .0129 & .0000 & .2141 \\
13 & .0000 & .0000 & -.0376 & 8.8587 \\
14 & -.7217 & .0083 & .0000 &
\end{tabular}
168.1632

$\begin{array}{llllll}15 & .0000 & .0000 & .2387 & -56.2774\end{array}$

$\begin{array}{lllll}16 & .0180 & .0962 & .0000 & -26.9201\end{array}$

$\begin{array}{lllll}17 & .0000 & .0000 & -.4807\end{array}$

113.3036

$\begin{array}{rrrrr}18 & .0000 & .0000 & -.1557 & 36.7008 \\ 19 & -.4188 & .1290 & .0000 & 68.2999 \\ 20 & -.0164 & .0529 & .0000 & -8.6214 \\ 21 & .0179 & .8629 & .0000 & - \\ 07.6309 & & & & \end{array}$


Ground to excited state transition densities written to RWF 633

Excitation energies and oscillator strengths:

Excited State 1: Singlet-B $3.2030 \mathrm{eV}$ $387.09 \mathrm{~nm} \mathrm{f}=0.0690$

$93->102 \quad .20408$

$101->102 \quad .64746$

This state for optimization and/or second-order correction.

Copying the excited state density for this state as the 1-particle RhoCI densit y.

Excited State 2: Singlet-A $3.9916 \mathrm{eV}$ $310.61 \mathrm{~nm} \mathrm{f}=0.0004$

$$
\begin{array}{ll}
92->102 & .55092 \\
92->108 & .20664 \\
92->116 & .16004 \\
95->102 & .21297 \\
97->102 & -.11090
\end{array}
$$

Excited State 3: Singlet-A $5.0702 \mathrm{eV}$ $244.54 \mathrm{~nm} \mathrm{f}=0.6973$

$$
\begin{array}{ll}
100->102 & .61951 \\
101->103 & .21760
\end{array}
$$

Excited State 4: Singlet-B $5.7477 \mathrm{eV}$ $215.71 \mathrm{~nm} \mathrm{f}=0.1365$

$93->102 \quad .11246$

$95->103 \quad-.14655$

$96->102 \quad-.33641$

$97->104 \quad-.10887$

$98->102 \quad .38241$

$99->103 \quad-.11339$

$$
\begin{array}{rr}
100->103 & -.14149 \\
100->104 & .10863 \\
101->105 & -.16199
\end{array}
$$

Excited State 5: Singlet-A $5.7879 \mathrm{eV}$ $214.21 \mathrm{~nm} \mathrm{f}=0.0047$

$$
94->103 \quad-.19110
$$

$94->107 \quad .10682$

$95->105 \quad .12223$

96 ->104 -.12999 


$$
\begin{array}{cc}
96->109 & .10937 \\
97->102 & .20462 \\
97->106 & -.11214 \\
98->103 & -.14327 \\
99->102 & .38757 \\
99->108 & -.12438 \\
100->105 & .15627 \\
100->106 & .11926 \\
101->104 & -.21535
\end{array}
$$

Excited State 6: Singlet-B $5.8591 \mathrm{eV}$ $211.61 \mathrm{~nm} \mathrm{f}=0.1575$

$$
\begin{array}{cc}
94->102 & .25556 \\
94->105 & .12044 \\
95->104 & .10280 \\
96->102 & .35855 \\
96->110 & .10219 \\
97->103 & -.17995 \\
98->102 & .11329 \\
99->103 & -.19073 \\
100->104 & .18391 \\
101->105 & -.11110 \\
101->106 & -.18112
\end{array}
$$

Excited State 7: Singlet-A $5.8841 \mathrm{eV}$ $210.71 \mathrm{~nm} \mathrm{f}=0.0016$

$$
\begin{array}{cc}
94->103 & -.12227 \\
95->105 & .11111 \\
96->104 & -.11176 \\
97->102 & .45254 \\
97->108 & -.10478 \\
98->103 & .15017 \\
98->107 & .17243 \\
99->102 & -.17154 \\
99->106 & -.17032 \\
100->110 & -.15496 \\
101->109 & .18361
\end{array}
$$

Excited State 8: Singlet-B $5.9646 \mathrm{eV}$ $207.87 \mathrm{~nm} \mathrm{f}=0.0015$

$$
\begin{array}{ll}
93->102 & .14133 \\
94->102 & .24933 \\
95->103 & -.15337 \\
96->102 & -.14427 \\
96->105 & -.15701 \\
97->103 & -.14214
\end{array}
$$

$$
\begin{array}{cc}
97->104 & -.12274 \\
97->107 & -.11938 \\
98->102 & -.19875 \\
98->106 & -.19663 \\
99->104 & -.12399 \\
99->107 & .15829 \\
100->103 & -.10322 \\
100->109 & -.20199 \\
101->108 & .18082 \\
101->110 & .16260
\end{array}
$$

Excited State 9: Singlet-B $6.0262 \mathrm{eV}$

$$
\begin{array}{cc}
205.74 \mathrm{~nm} & \mathrm{f}=0.0602 \\
93->102 & .26526 \\
95->104 & -.13302 \\
96->102 & .11634 \\
96->106 & .14050 \\
97->109 & .11418 \\
98->105 & -.14714 \\
99->107 & -.12001 \\
100->103 & -.25750 \\
100->104 & -.12556 \\
100->107 & -.11453 \\
101->106 & -.11211 \\
101->108 & .23575 \\
101->110 & -.12894
\end{array}
$$

Excited State 10: Singlet-A 6.1040

$\mathrm{eV} 203.12 \mathrm{~nm} \mathrm{f}=0.0205$

$$
\begin{array}{ll}
92->102 & -.12589 \\
94->103 & -.10550 \\
94->104 & -.14578 \\
95->102 & .37183 \\
96->103 & .18898 \\
97->102 & -.13868 \\
97->105 & -.13365 \\
97->106 & -.10300 \\
98->107 & .14638 \\
98->109 & .11701 \\
99->110 & .13438 \\
100->106 & -.13045 \\
100->110 & -.12149 \\
101->107 & -.11730 \\
101->109 & .15736
\end{array}
$$


Excited State 11: Singlet-A 6.1776 $\mathrm{eV} 200.70 \mathrm{~nm} \mathrm{f}=0.0300$

$$
\begin{array}{cc}
96->107 & -.10920 \\
99->105 & -.11493 \\
100->102 & -.26116 \\
100->108 & -.22378 \\
101->103 & .46053 \\
101->107 & .14312
\end{array}
$$

Excited State 12: Singlet-B 6.5063 eV $190.56 \mathrm{~nm} \mathrm{f}=0.0035$

$$
\begin{array}{cc}
90->102 & -.10050 \\
94->105 & -.13195 \\
95->104 & -.11993 \\
96->102 & .28931 \\
96->108 & .17037 \\
97->104 & -.14088 \\
98->102 & -.13901 \\
98->110 & .13305 \\
99->109 & .15157 \\
100->103 & -.10594 \\
100->107 & .23501 \\
101->105 & -.15816 \\
101->106 & .28682
\end{array}
$$

Excited State 13: Singlet-A 6.5613

eV $188.96 \mathrm{~nm} \mathrm{f}=0.0062$

$$
\begin{array}{cc}
94->104 & -.14410 \\
95->102 & -.15926 \\
95->108 & -.10948 \\
96->103 & .10008 \\
97->102 & .28328 \\
97->105 & -.12134 \\
97->108 & .11877 \\
98->109 & .15486 \\
99->110 & .13927 \\
100->105 & .10822 \\
100->106 & -.18860 \\
101->103 & .15364 \\
101->104 & .11817 \\
101->107 & -.30317
\end{array}
$$

Excited State 14: Singlet-B 6.7192 eV $184.52 \mathrm{~nm} \mathrm{f}=0.0992$

$93->102 \quad .15309$

$96->102 \quad .16733$

$$
\begin{array}{cc}
97->109 & -.11105 \\
98->102 & .45493 \\
98->108 & .13432 \\
100->104 & -.16806 \\
100->109 & -.11194 \\
101->105 & .19053 \\
101->106 & .16355 \\
101->110 & .11515
\end{array}
$$

Excited State 15: Singlet-A 6.7254

eV $184.35 \mathrm{~nm} \mathrm{f}=0.1545$

$$
\begin{array}{ll}
96->109 & -.10499 \\
99->102 & .52323 \\
99->108 & .11908 \\
100->105 & -.16548 \\
100->106 & -.11342 \\
101->104 & .25873 \\
101->109 & .14903
\end{array}
$$

Excited State 16: Singlet-B 6.8800 $\mathrm{eV} 180.21 \mathrm{~nm} \mathrm{f}=0.0304$

$$
\begin{array}{cc}
90->102 & .10039 \\
93->102 & .40483 \\
94->102 & .22924 \\
96->102 & -.15083 \\
97->104 & .10933 \\
98->102 & -.20703 \\
98->106 & .10752 \\
99->104 & .13593 \\
100->109 & .10529 \\
101->102 & -.14176 \\
101->108 & -.17367
\end{array}
$$

Excited State 17: Singlet-A 6.9945 eV $177.26 \mathrm{~nm} \mathrm{f}=0.8882$

$$
\begin{array}{cc}
93->103 & .18231 \\
94->104 & .21083 \\
94->109 & -.12858 \\
95->106 & .11040 \\
95->110 & -.11779 \\
96->107 & .10258 \\
97->110 & -.16588 \\
98->104 & .17882 \\
98->109 & .15643 \\
99->105 & .23598 \\
101->103 & .34513
\end{array}
$$




$\begin{array}{cc}101->115 & .10417 \\ \text { Excited State } & 18: \text { Singlet-A } \\ \mathrm{eV} 175.69 \mathrm{~nm} & \mathrm{f}=0.0348 \\ 94->104 & .10111 \\ 95->102 & .45160 \\ 95->105 & -.10164 \\ 96->104 & .13533 \\ 97->102 & .28187 \\ 99->106 & .11120 \\ 100->105 & -.10193 \\ 100->110 & .13764 \\ 101->104 & .15354 \\ 101->109 & -.15539\end{array}$

Excited State 19: Singlet-B 7.2236 $\mathrm{eV} 171.64 \mathrm{~nm} \mathrm{f}=0.1355$

$$
\begin{array}{cc}
93->102 & -.29164 \\
94->102 & .49517 \\
95->104 & -.13331 \\
100->104 & -.17267 \\
101->105 & .14134 \\
101->110 & -.13370
\end{array}
$$

Excited State 20: Singlet-B 7.4260 eV $166.96 \mathrm{~nm} \mathrm{f}=0.1575$

$$
\begin{array}{cc}
91->103 & -.10157 \\
93->108 & .10994 \\
94->110 & -.14267 \\
97->109 & -.15796 \\
98->105 & .17225 \\
98->108 & .12016 \\
98->110 & .12231 \\
99->104 & .23073 \\
99->109 & .12707 \\
100->103 & -.15499 \\
101->105 & -.14443 \\
101->106 & -.10251 \\
101->108 & .36641
\end{array}
$$

Excited State 21: Singlet-B 7.6123

eV $162.87 \mathrm{~nm} \mathrm{f}=1.7490$

$$
\begin{array}{ll}
93->106 & -.11904 \\
94->105 & -.10919 \\
94->106 & -.10990 \\
95->104 & -.11210
\end{array}
$$

$$
\begin{array}{cc}
97->104 & -.19833 \\
98->110 & .16609 \\
99->109 & .19315 \\
101->105 & .27298 \\
101->106 & -.40163
\end{array}
$$

Excited State 22: Singlet-A 7.6805

$\mathrm{eV} 161.43 \mathrm{~nm} \mathrm{f}=0.0245$

$$
\begin{array}{cc}
87->102 & .11350 \\
91->102 & .36291 \\
94->103 & -.11607 \\
98->107 & -.11247 \\
98->109 & -.10097 \\
99->108 & -.17330 \\
101->104 & .30026 \\
101->107 & -.19640
\end{array}
$$

Excited State 23: Singlet-A 7.8562 eV $157.82 \mathrm{~nm} \mathrm{f}=0.6455$

$$
\begin{array}{ll}
87->102 & .11158 \\
91->102 & .33045 \\
94->103 & .15833 \\
94->107 & -.11886 \\
97->106 & .16292 \\
98->103 & .10386 \\
99->108 & .10675 \\
101->104 & -.39722 \\
101->107 & -.14926
\end{array}
$$

Excited State 24: Singlet-B 7.8796 eV $157.35 \mathrm{~nm} \mathrm{f}=0.5536$

$$
\begin{array}{cc}
90->102 & .10869 \\
94->102 & -.12849 \\
94->106 & -.12602 \\
97->103 & -.17195 \\
97->107 & .16391 \\
98->106 & .10134 \\
98->108 & -.13400 \\
99->103 & -.29329 \\
100->103 & .10141 \\
100->104 & -.17340 \\
101->105 & .21997 \\
101->106 & .16152 \\
101->108 & .26651
\end{array}
$$


Excited State 25: Singlet-A 7.9388 $\mathrm{eV} 156.18 \mathrm{~nm} \mathrm{f}=0.0026$

$$
\begin{array}{cc}
91->102 & .25891 \\
93->107 & .14798 \\
95->102 & -.11725 \\
96->103 & .13138 \\
98->103 & -.14546 \\
98->109 & .13656 \\
99->106 & .10994 \\
99->110 & .10635 \\
101->107 & .42606
\end{array}
$$

Excited State 26: Singlet-B 7.9590

$$
\text { eV } 155.78 \mathrm{~nm} \mathrm{f}=0.2688
$$

$$
\begin{array}{cc}
94->105 & -.15086 \\
95->103 & .13702 \\
95->104 & -.12188 \\
99->104 & -.12711 \\
100->103 & .40765 \\
101->102 & -.11518 \\
101->105 & -.27771 \\
101->108 & .16685 \\
101->110 & -.10893
\end{array}
$$

Excited State 27: Singlet-A 8.1637 eV $151.87 \mathrm{~nm} \mathrm{f}=0.5411$

$$
\begin{array}{ll}
91->102 & -.17497 \\
94->103 & .12999 \\
98->107 & -.24720 \\
99->106 & .17063 \\
99->108 & -.12463 \\
100->105 & -.11650 \\
100->110 & -.14947 \\
101->104 & -.10335 \\
101->109 & .42331
\end{array}
$$

Excited State 28: Singlet-B 8.1715

$$
\text { eV } 151.73 \mathrm{~nm} \mathrm{f}=0.2580
$$

$$
\begin{array}{cc}
94->108 & .12000 \\
95->103 & .13200 \\
97->103 & .21651 \\
98->106 & .21774 \\
99->107 & -.21639 \\
100->103 & .10778 \\
100->104 & .11064 \\
100->107 & -.10484
\end{array}
$$

$$
\begin{array}{ll}
100->109 & -.15434 \\
101->108 & .16281 \\
101->110 & .32951
\end{array}
$$

Excited State 29: Singlet-B 8.2372

eV $150.52 \mathrm{~nm} \mathrm{f}=0.3111$

$$
\begin{array}{cc}
66->102 & .12690 \\
73->102 & .13033 \\
77->102 & .21812 \\
79->102 & .13561 \\
81->102 & -.13876 \\
88->102 & .20550 \\
90->102 & .29785 \\
92->103 & .11390 \\
93->102 & -.12563 \\
95->103 & .17350 \\
100->103 & -.13891 \\
101->105 & -.11691 \\
101->110 & .15381
\end{array}
$$

Excited State 30: Singlet-A 8.4496

$\mathrm{eV} 146.73 \mathrm{~nm} \mathrm{f}=0.3694$

$$
\begin{array}{cc}
72->102 & -.10007 \\
80->102 & .10265 \\
92->102 & .10384 \\
93->104 & .14128 \\
94->103 & .13897 \\
94->104 & .11184 \\
95->105 & .10672 \\
95->110 & -.12693 \\
96->104 & -.11402 \\
96->109 & .10599 \\
97->105 & -.17662 \\
98->104 & .16579 \\
99->106 & .13325 \\
99->108 & .10571 \\
100->105 & .24766 \\
100->108 & -.15013 \\
101->104 & .18303
\end{array}
$$

